\title{
Lateral impacts correlate with falx cerebri displacement and corpus callosum trauma in sports-related concussions
}

\author{
Fidel Hernandez ${ }^{1} \cdot$ Chiara Giordano $^{2} \cdot$ Maged Goubran $^{3} \cdot$ Sherveen Parivash $^{3} \cdot$ Gerald Grant $^{4,5} \cdot$ Michael Zeineh $^{3}$. \\ David Camarillo ${ }^{1,2}$
}

Received: 14 June 2018 / Accepted: 5 December 2018 / Published online: 12 March 2019

(C) The Author(s) 2019

\begin{abstract}
Corpus callosum trauma has long been implicated in mild traumatic brain injury (mTBI), yet the mechanism by which forces penetrate this structure is unknown. We investigated the hypothesis that coronal and horizontal rotations produce motion of the falx cerebri that damages the corpus callosum. We analyzed previously published head kinematics of 115 sports impacts (2 diagnosed mTBI) measured with instrumented mouthguards and used finite element (FE) simulations to correlate falx displacement with corpus callosum deformation. Peak coronal accelerations were larger in impacts with mTBI (8592 rad/ $\mathrm{s}^{2}$ avg.) than those without (1412 rad/ $\mathrm{s}^{2}$ avg.). From FE simulations, coronal acceleration was strongly correlated with deep lateral motion of the falx center $(r=0.85)$, while horizontal acceleration was correlated with deep lateral motion of the falx periphery $(r>0.78)$. Larger lateral displacement at the falx center and periphery was correlated with higher tract-oriented strains in the corpus callosum body $(r=0.91)$ and genu/splenium $(r>0.72)$, respectively. The relationship between the corpus callosum and falx was unique: removing the falx from the FE model halved peak strains in the corpus callosum from 35\% to $17 \%$. Consistent with model results, we found indications of corpus callosum trauma in diffusion tensor imaging of the mTBI athletes. For a measured alteration of consciousness, depressed fractional anisotropy and increased mean diffusivity indicated possible damage to the mid-posterior corpus callosum. Our results suggest that the corpus callosum may be sensitive to coronal and horizontal rotations because they drive lateral motion of a relatively stiff membrane, the falx, in the direction of commissural fibers below.
\end{abstract}

Keywords Corpus callosum trauma $\cdot$ Mild traumatic brain injury $\cdot$ Finite element method $\cdot$ Instrumented mouthguard

\section{Introduction}

Mild traumatic brain injury (mTBI) is a growing health threat that is notoriously underreported, difficult to screen, and poorly understood. In 2004, mTBI affected 6/1000 people worldwide and comprised $70-90 \%$ of all reported TBI

Fidel Hernandez, Chiara Giordano, and Maged Goubran: Co-first authors

Michael Zeineh and David Camarillo: Co-senior authors

Electronic supplementary material The online version of this article (https://doi.org/10.1007/s10237-018-01106-0) contains supplementary material, which is available to authorized users.

David Camarillo

dcamarillo@stanford.edu

Extended author information available on the last page of the article
(Cassidy et al. 2004). In subsequent years, reported mTBI incidence rates grew by 14-26\% in high school sports (Lincoln et al. 2011; Rosenthal et al. 2014), 15-25\% in the military (Ivins 2010; Cameron et al. 2012), and 7\% in emergency department visits (Coronado et al. 2012). In sports and recreation, evidence of long-term neurological impairment in athletes suffering repeat mTBI (Guskiewicz et al. 2003; Omalu et al. 2005, 2006; Maroon et al. 2015; Montenigro et al. 2017) suggests a pressing need to improve current screening methods by uncovering the mechanism of injury. In particular, the mechanism by which skull accelerations produce neurological impairment is not completely understood. (A theoretical model is presented in Fig. 1.)

Skull acceleration measurements have helped inform our understanding of brain tolerance to trauma. Early efforts to measure skull accelerations have leveraged sensor devices mounted to headbands, ear plugs, helmets, bite bars, and mouthguards (Naunheim et al. 2003; Knox 2004; Funk et al. 
2009; Rowson et al. 2011; Camarillo et al. 2013; Bartsch et al. 2014). While the accuracy of these devices is still a subject of investigation (Jadischke et al. 2013; Kuo et al. 2016; Siegmund et al. 2016; Wu et al. 2016), criteria derived from skull measurements have been found to predict mTBI (Ommaya et al. 1966; Rowson et al. 2012; Hernandez et al. 2015a; Hernandez and Camarillo 2018). In particular, rotational measurements have been shown to improve injury prediction (Hernandez et al. 2015a). However, the sensitivity of the brain to rotation of the skull is thought to vary by the direction of rotation. Coronal rotations produce the longest period of post-traumatic loss of consciousness and the most severe axonal damage in primates (Gennarelli et al. 1982, 1987). Horizontal and sagittal rotations produce the greatest axonal injuries in swine, but interspecies differences in brain-skull anatomy and relative brainstem orientation may explain these results (Smith et al. 2000; Browne et al. 2011; Eucker et al. 2011). In computational simulations, horizontal plane rotations produce the largest overall brain deformations (Weaver et al. 2012). Given the complexity of skull rotation, mapping skull accelerations to brain trauma requires that we understand which brain deformations and structures are most clinically relevant.

In the last decades, laboratory and computational models have been used to estimate brain deformation in mTBI. In particular, finite element (FE) models have been used to investigate brain deformations that are sensitive to severe and arbitrary skull acceleration inputs (Kleiven 2006; Takhounts et al. 2008; Ji et al. 2015; Sullivan et al. 2015). Principal-direction tissue strain is most commonly drawn from these models to assess injury risk, reaching 10-45\% in sports mTBI impacts (Kleiven 2007). However, recent studies suggest that white matter tract-direction tissue strain is more anatomically and clinically relevant than principaldirection strain (Giordano and Kleiven 2014; Ji et al. 2015; Sullivan et al. 2015) and may represent a good injury predictor to identify which brain structures are most affected in mTBI.

Neurological deficits from tissue strain are also thought to depend on the affected brain structure. The corpus callosum, a white matter structure that joins both brain hemispheres above the lateral ventricles, may undergo high strain during mTBI (Giordano and Kleiven 2014; Hernandez et al. 2015a), and callosal injury is thought to affect perception, speech, orientation, and reaction (Ungerleider and Mishkin 1982; Gazzaniga et al. 1988; Arenth et al. 2014). Another periventricular structure, the thalamus, has been implicated in mTBI and suspected to affect consciousness when damaged (Schaltenbrand 1975; Giordano and Kleiven 2014). Periventricular damage, broadly speaking, is a signature of chronic traumatic encephalopathy (Omalu et al. 2006; Smith 2015), yet the mechanism by which deformation penetrates this region is unknown. While prior work implicates coronal rotation (Gennarelli et al. 1982, 1987), horizontal rotation and corpus callosum strain in mTBI, it is unclear if and how these are related. Hypotheses connecting head biomechanics to injury diagnosis are derived from disparate data sets (human, animal, analytic, physical, and computational) and do not provide a unifying understanding of the mechanism of injury.

One structure that is thought to affect the corpus callosum during skull rotation is the falx cerebri, a rigid membrane in the longitudinal fissure that is located just above the corpus callosum (Fig. 2). Physical models of the baboon brain have found that the falx protects the corpus callosum from large shear strains (Fig. 2a) (Margulies

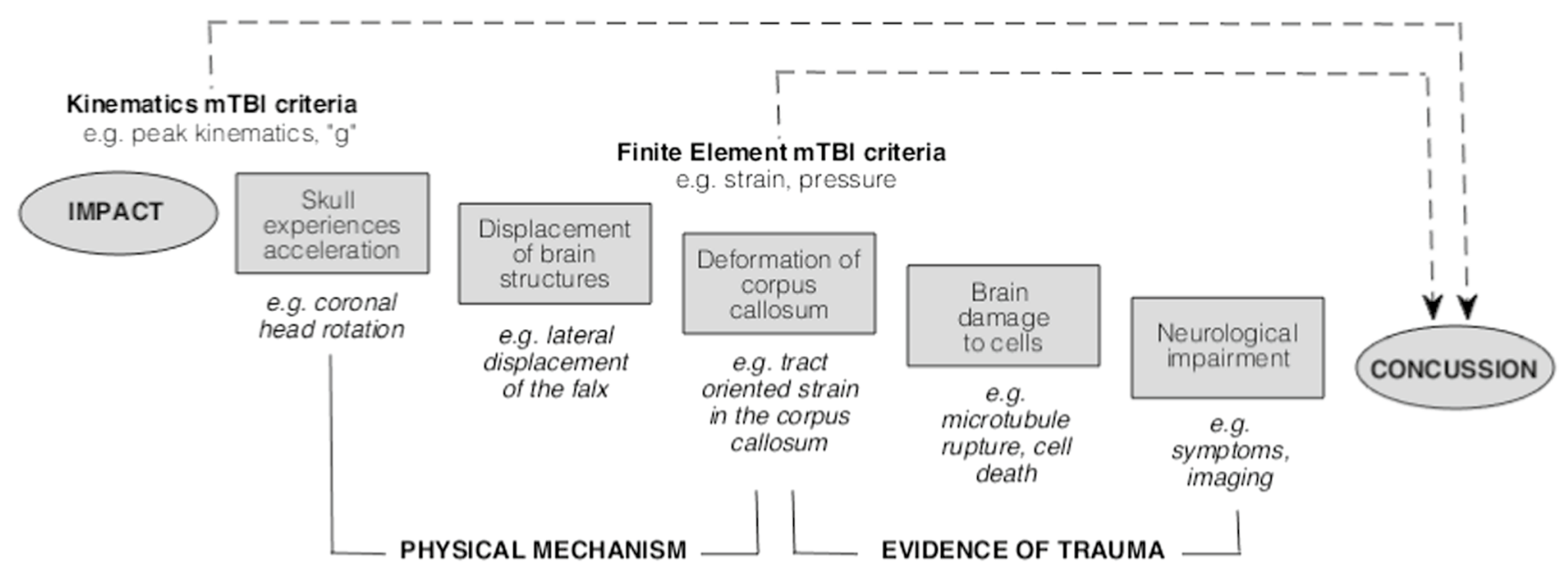

Fig. 1 Theoretical model of how head accelerations are related to neurological impairment in mTBI. Head accelerations and brain deformation are often used to describe mTBI diagnosis. However, it may be just as necessary to understand the mechanisms by which accelerations produce brain tissue deformation, and by which tissue deformation produces neurological impairment. In this study, we propose important, connected factors that may have produced diagnosed symptoms from mTBI impacts 
et al. 1990). In contrast, 2D computational models of the human brain found a softer falx, or no falx at all, actually maintained or decreased strains in the corpus callosum (Fig. 2b) (Nishimoto and Murakami 1998; Li et al. 2007). In another study, it was hypothesized that the falx induces strain in the corpus callosum during coronal rotation by impinging on the motion of the trailing hemisphere and thereby creating drift along the sagittal midline (Fig. 2c) (Smith et al. 2000). Using a 3D detailed FE model of the human head, a recent study found that the presence of the falx causes a steep stiffness gradient at the inferior loose end of the membrane during predominantly horizontal rotations, which may induce a strain concentration in the corpus callosum (Ho et al. 2017). Furthermore, insights on the role of the falx in propagating deformation to deep brain structures in living humans come from recent studies using tagged MRI to measure brain displacements and deformations due to mild translational or rotational accelerations (Bayly et al. 2005; Sabet et al. 2008; Feng et al. 2010). In particular during coronal mild impacts in vivo (Sabet et al. 2008), symmetry and anti-symmetry were observed in brain shear strain fields indicated that the falx interacts with the surrounding brain tissue and induces strain at these sites. The medial longitudinal fissure appeared to isolate the hemispheres of the brain superior to the corpus callosum. The specific roles of the falx
A The presence of the falx protects the brain from large strains

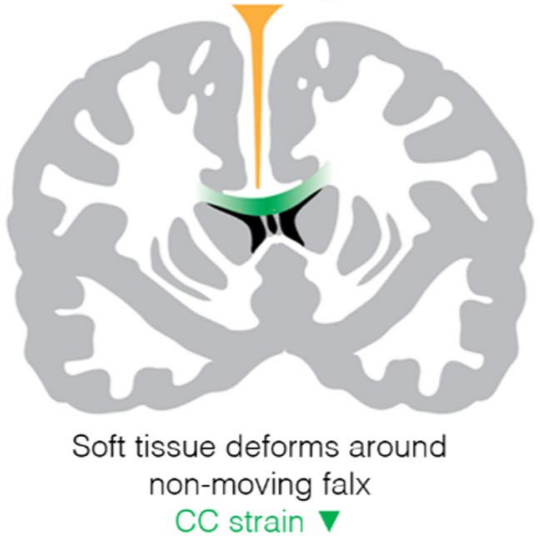

C The falx impinges the motion
of the trailing hemisphere

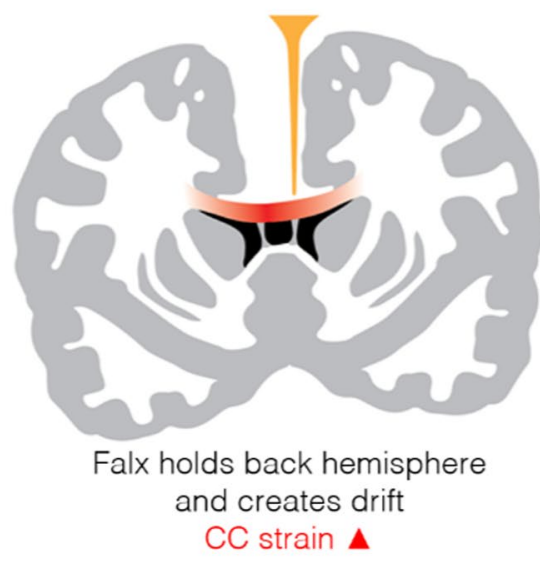

Fig. 2 Hypothesized mechanism of interaction between the falx cerebri and the midsagittal region of the brain from previously published studies and the current study. The upward-pointing triangle and the downward-pointing triangle indicate an increase and a decrease in corpus callosum strain, respectively, due to brain tissue interaction with the falx. a Margulies et al. (1990) proposed that the presence of the falx protected the corpus callosum from large shear strain. b In

\section{B The falx generates a stiffness gradient.}

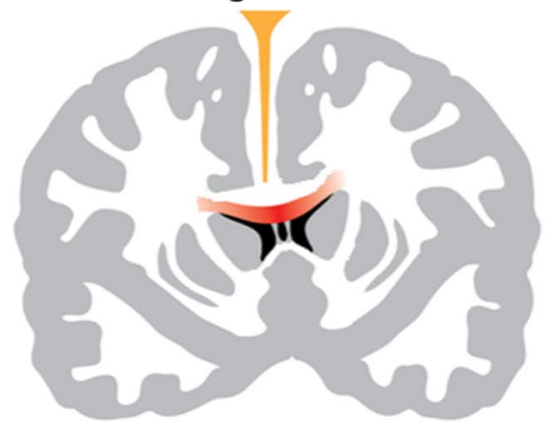

Rigid falx provides a lateral constrain creating strain in deep brain tissue $\mathrm{CC}$ strain $\boldsymbol{\Delta}$

D Present study - Rotation produces
lateral displacement of the falx

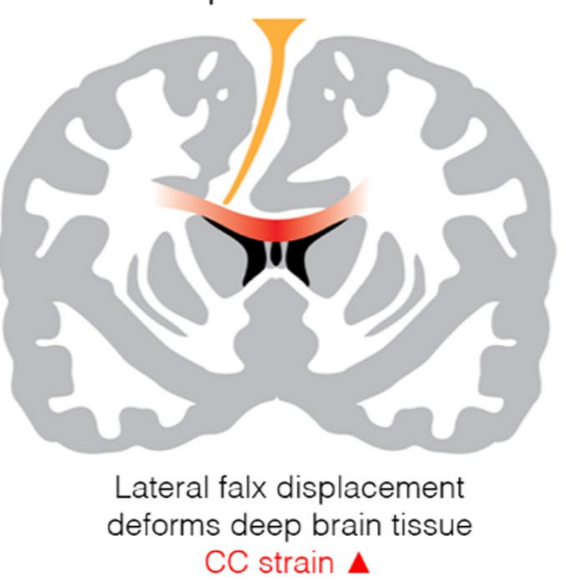

contrast, from computational modeling (Nishimoto and Murakami 1998; Li et al. 2007), it was suggested that the presence of a rigid falx increases strains in the corpus callosum. c Smith et al. (2000) hypothesized that the falx impinges the trailing hemisphere and produces a midline shift that affects the corpus callosum. d Our hypothesis is that coronal and horizontal rotation produces motion of the falx cerebri that in turn strains the corpus callosum below it and damages it 
are currently unknown and remain topics of great interest in TBI research.

This study investigates the mechanism by which skull movement produces brain deformation that penetrates deep brain structures. In particular, we studied the relationships between head motion, falx displacement, and corpus callosum trauma. Using previously published measurements of human head translation and rotation during mTBI (Hernandez et al. 2015a), we linked skull rotation to brain structure deformation using a finite element head model (Kleiven 2007). To provide evidence of trauma in diagnosed concussions, we used diffusion tensor imaging (DTI) and performed region of interest analyses. In the last decade, DTI has been increasingly used to identify white matter injury in mTBI and to determine possible association between diffusion measures and cognitive, behavioral, and neuropsychiatric disorders in mTBI (Niogi and Mukherjee 2010; Shenton et al. 2012; Eierud et al. 2014). Analyzing and combining biomechanical and imaging findings, we propose the hypothesis that coronal and horizontal rotation produces motion of the falx cerebri that in turn strains and damages the underlying corpus callosum (Fig. 2d).

\section{Methods}

\subsection{Measurement of sports head impacts}

Six-degree-of-freedom (6DOF) measurements of 115 sports head impacts were analyzed in this study: translation in the anterior-posterior, left-right, and superior-inferior directions and rotation in the coronal, sagittal, and horizontal planes. At Stanford, instrumented mouthguards measured 6DOF head accelerations during over 500 videoconfirmed head impacts in football (Fig. 3a) (Hernandez et al. 2015a). Of these, 2 head impacts immediately preceded an mTBI diagnosis. One mTBI resulted in the subject suffering alteration of consciousness (AOC), while the other was milder and self-reported (SR). Along with both injuries, 113 of the other (sub-concussive) impacts were selected for further analysis: 50 selected at random, 58 selected because they exceeded either injury in any translational or rotational acceleration component, and 5 selected because they were sustained by the self-reported mTBI subject on the same day (and prior to) his injury. This subset was therefore enriched for high acceleration impacts that are the most difficult to distinguish from the mTBI cases. The dataset included impacts in all directions at different locations. The kinematic accuracy of these instrumented mouthguards has been previously validated in dummy head, in vivo, and ex vivo testing (Camarillo et al. 2013; Hernandez et al. 2015a; Kuo et al. 2016; Wu et al. 2016).

\subsection{Finite element modeling of the brain}

Estimates of brain tissue deformation for all Stanford head impacts were obtained from simulations using the KTH finite element (FE) model (KTH Royal Institute of Technology, Stockholm, Sweden) (Kleiven 2006, 2007). This model includes the brain, skull, scalp, meninges, cerebrospinal fluid (CSF), and 11 pairs of bridging veins (Fig. 3b). Skull acceleration was prescribed to follow the measured 6DOF head accelerations, and ensuing brain deformation was observed. The brain was modeled as a hyper-viscoelastic material and divided into seven primary structures: (1) corpus callosum, (2) brainstem, (3) cerebellum, (4) cerebral gray matter (cortex), (5) cerebral white matter, (6) midbrain,
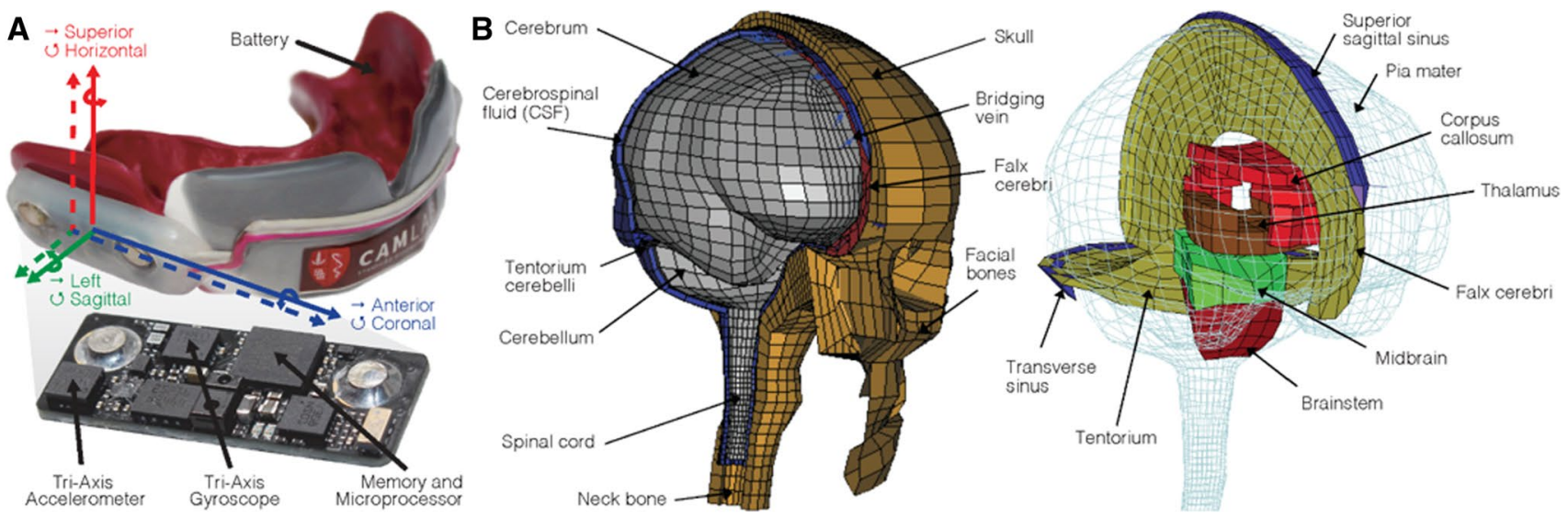

Fig. 3 Measurement of head biomechanics and simulation of brain deformation. a Head accelerations during impact were measured in six-degree-of-freedom (6DOF) measurements using instrumented mouthguards. Mouthguard measurements were used in a simulation consisting of a b finite element head model incorporating the scalp, skull, brain, cerebrospinal fluid, and bridging veins 
and (7) thalamus. The corpus callosum was further divided into thirds: the genu (anterior portion), the body (middle portion), and the splenium (posterior portion). An Ogden hyper-elastic constitutive law was used to model the brain and to account for large deformations of the tissue, with additional linear viscoelastic terms to account for the rate dependence of the tissue. The boundary condition between the dura and skull was tied. Between the brain and dura, a sliding interface was implemented that allowed tangential but not radial movement between the structures (given the incompressibility of the mostly water CSF). The determined brain geometry and material properties were compared to displacement data from cadaver head impact experiments where neutral density targets were inserted inside cadaver brains and tracked using high-speed biplanar X-ray during impacts (Hardy 2001; Hardy et al. 2008). Moreover, to increase the confidence in predicting tissue strain in living subjects, we compared the model strain predictions on subinjury strains measured using tagged MRI in frontal impact (Feng et al. 2010). More details about the comparison can be found in supplementary material S1.

Modifications to FE model were tested to investigate the effect of various brain structures on resulting brain tissue deformations. Most of these modifications pertained to the falx cerebri, which was hypothesized to generate strain in the corpus callosum. In the original model, the falx cerebri is a 1.5-mm-thick linear elastic shell with a Young's modulus of 31.5 MPa (Kleiven 2007). Since the falx is an infolding of the dura mater, it is rigidly attached (via tied contact in LS-DYNA) to the top of the skull along the sagittal midline, anteriorly at the crista galli and posteriorly to the upper surface of the tentorium. The falx is also surrounded by CSF along the lateral faces and inferior aspect just above the corpus callosum (Fig. 3b). Along the same lines, the falx has the same previously described sliding interface with the cerebral hemispheres on either side and with the corpus callosum below. Simulations of all Stanford impacts were performed on a version of the model with the falx, and all other meningeal shell layers removed from inside the longitudinal fissure of the brain, leaving only the CSF. Simulations of the alteration of consciousness (AOC) mTBI were also performed on a model with only the falx removed while the pia mater was in place, and on a model with the entire brain fissure closed (filled in with hyper-viscoelastic brain material connecting the two hemispheres).

Additional simulations of only the AOC mTBI were run for various falx Young's moduli. We decreased the falx Young's modulus to $31.5 \mathrm{kPa}$ (just below the approximate stiffness of the brain, which is $66.7 \mathrm{kPa}$ in the FE model) and increased the falx stiffness up to $10 \mathrm{GPa}$ (above the approximate stiffness of diploe, or spongy skull bone, which is $1 \mathrm{GPa}$ in the FE model). We run a total of 24 simulations for the following values of falx Young's moduli: $3.15 \times 10^{4}$
$\mathrm{Pa}, 3.15 \times 10^{5} \mathrm{~Pa}, 3.15 \times 10^{6} \mathrm{~Pa}, 3.15 \times 10^{7} \mathrm{~Pa}, 3.15 \times 10^{8} \mathrm{~Pa}$, $3.15 \times 10^{9} \mathrm{~Pa}, 5 \times 10^{4} \mathrm{~Pa}, 6.67 \times 10^{4} \mathrm{~Pa}$ (brain-like), $1 \times 10^{5}$ $\mathrm{Pa}, 1 \times 10^{6} \mathrm{~Pa}, 1 \times 10^{7} \mathrm{~Pa}, 1 \times 10^{8} \mathrm{~Pa}, 1 \times 10^{9} \mathrm{~Pa}$ (spongy bone-like), $1 \times 10^{10} \mathrm{~Pa}$ (compact bone-like), $2 \times 10^{7} \mathrm{~Pa}$, $3 \times 10^{7} \mathrm{~Pa}, 4 \times 10^{7} \mathrm{~Pa}, 5 \times 10^{7} \mathrm{~Pa}, 2 \times 10^{8} \mathrm{~Pa}, 5 \times 10^{8} \mathrm{~Pa}$, $1.5 \times 10^{9} \mathrm{~Pa}, 2 \times 10^{9} \mathrm{~Pa}, 5 \times 10^{9} \mathrm{~Pa}$, and $8 \times 10^{9} \mathrm{~Pa}$. To investigate alternative hypotheses involving other structures near the corpus callosum, the kinematics of the AOC mTBI were imposed on a model without ventricles. The presence of an incompressible fluid like the ventricles could potentially create a stress concentration and produce a strain gradient close to the corpus callosum. Finally, because another alternative hypothesis is that the presence of the septum pellucidum affects corpus callosum strain, we modified the model to include a septum pellucidum as a linear elastic midline shell attaching superiorly to the genu of the corpus callosum and inferiorly to the thalamus. We used an approximate thickness of $2 \mathrm{~mm}$, consistent with the 1.5-3 mm range observed in humans (Pendergrass and Hodes 1935). We ran two simulations of the AOC mTBI for a wide span of septum pellucidum stiffnesses (Young's modulus $=66.7 \mathrm{kPa}$ and $1 \mathrm{GPa}$ ).

To investigate directional dependence, we performed simulations of the Stanford head impacts with isolated components of the acceleration measurements. We ran all 115 impacts an additional eight times with a component (or group of) skull accelerations and the remaining zeroed out: all rotational velocities (3DOF), only coronal, only sagittal, only horizontal, all translational accelerations (3DOF), only anterior-posterior, only left-right, and only superior-inferior.

\subsection{Computation of brain mechanics and strain}

In all simulations, we analyzed the deformation of brain tissue in the primary direction of fiber tracts using a previously published technique (Giordano et al. 2014). Average tractography was determined from DTI of 81 healthy adult subjects. After scaling and registration among the images, the principal direction of diffusion (oriented along the fiber tract) and the degree of fractional anisotropy (FA) were averaged across all subjects. This average DTI volume was referenced against the FE model. For each element in the FE model, corresponding voxels in the DTI were selected and their average direction and FA were computed. In postprocessing of the simulations, the Green-Lagrange strain tensor was extracted and then projected in this average tract direction to determine the tract strain in each individual element: the largest eigenvalue of the tensor corresponded to the principal strain. Before performing this projection, the strain tensor was transformed to the inertial skull-fixed frame using a least-squares approximation (Arun et al. 1987). Tract directions were approximately superior-inferior in the parietal lobe, corona radiata and thalamus, lateral 
in the corpus callosum, and diagonal $\left(45^{\circ}\right)$ in the frontal lobe. Tract strain is only reported for elements with FA $>0.2$ (which includes most white matter and excludes most gray matter). When we replaced ventricles with brain tissue in the simulation, we did not compute tract strain in the ventricular region because there were no corresponding tracts in the reference DTI volume. Strain rate and strain $\times$ strain rate were computed using five-point stencil derivative of element strains over time. Peaks in strain, strain rate, and strain $\times$ strain rate were extracted over time, over the entire brain, and in subsets of structures. When extracting brain strains, nodal averaging (the default setting on LS-PrePost) was not used since the nodal value of the tract-oriented strain was not defined (projection was operated along the principal direction of fibers in an element).

In addition to brain tissue strain, we tracked the motion of the falx cerebri during all impacts. In the midsagittal plane, we tracked the location of 11 nodes on the falx, 6 distal (peripheral) and 5 proximal (deep) spaced evenly from anterior to posterior, as well as their closest neighbors on both of the cerebral hemispheres (Fig. 4). In the midcoronal plane, we tracked the displacement of 6 falx nodes running superior-inferior as well as their closest neighbors on either hemisphere (Fig. 4). Node locations were extracted in the ground-fixed (inertial) frame and re-expressed in an anatomically relevant skull-fixed frame (anterior-posterior, left-right, and superior-inferior) to quantify displacement from normal. The transformation between the skull-fixed and ground-fixed frames was defined using three planar skull nodes tracked for all impacts.

\subsection{Injury diagnosis and imaging}

Among the impacts measured with the Stanford mouthguard, two of these resulted in the diagnosis of mild TBI in two patients. Subject 1 was a 21-year-old male NCAA collegiate football player who sustained brief extensor posturing of his upper extremities and alteration of consciousness lasting approximately $2 \mathrm{~min}$. The subject was monitored for 3 days post-injury and was found to have an altered mental status along with post-concussive symptoms. However, his detailed neurological examination and 3T clinical brain MRI obtained within $24 \mathrm{~h}$ were both normal. Subject 1 had one prior mTBI with loss of consciousness at 21 years old and one prior mTBI without loss of consciousness at 19 years old, from which, in both cases, he recovered quickly with no significant post-concussive symptoms. Subject 2 was a 20-year-old male NCAA collegiate football player who sustained a concussion during practice. He did not lose consciousness, but he did self-report several post-concussive symptoms immediately following the impact including headache, poor concentration, and slowed reaction time. His detailed neurological examination and 3T brain MRI at $18 \mathrm{~h}$ post-injury were also normal. Subject 2 had no history of any chronic medical problems and did have one prior mTBI without loss of consciousness at 15 years old with no sequelae and no imaging done at that time.

Diffusion imaging was conducted for Stanford injuries both on the AOC mTBI at 1.5 years post-injury and on the SR mTBI subject at both 1-day post-injury and 2.5 months follow-up. We included in this analysis for comparison an additional cohort of six football players with no prior history of concussion. Controls were scanned at baseline, before

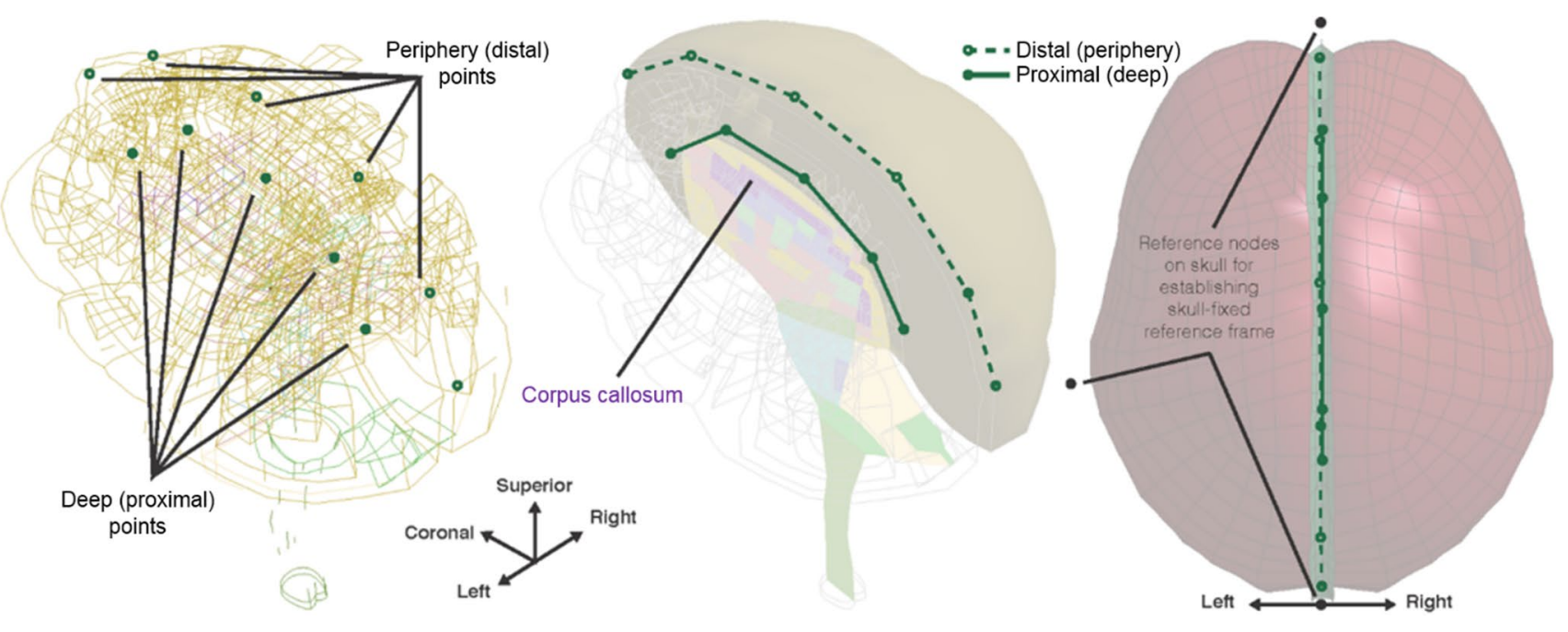

Fig. 4 Simulation of falx cerebri displacement. Displacement in the anterior-posterior, left-right (lateral), and superior-inferior directions were estimated for 11 nodes on the falx cerebri: 5 proximal (deep) to the brain center of gravity just above the corpus callosum and 6 distal along the boundary with the skull 
the beginning of the football season. Using a 3T MRI scanner (GE, Milwaukee, WI, USA, utilizing the same scanner for all subjects except the AOC, which used an identical GE scanner at a different physical location) and an eightchannel receiver head coil, we acquired multiple non-contrast sequences and here reported results on the images used for DTI analysis, which included whole-brain structural T1-weighted images (FSPGR BRAVO, $0.93 \times 0.93 \times 1 \mathrm{~mm}$, $5 \mathrm{~min}$ ) and DTI: 60 directions at $b=2500,30$ directions at $b=800 \mathrm{~s} / \mathrm{mm}^{2}, b_{0} \mathrm{~s}=9, \mathrm{TE}=81.6 \mathrm{~ms}, \mathrm{TR}=8500 \mathrm{~ms}$, matrix $=128 \times 128$, FOV $24 \mathrm{~cm}$, slice thickness $=2 \mathrm{~mm}$. Football participants in the study provided written informed consent in accordance with and under the approval of Stanford's Internal Review Board (IRB) and the Health Insurance Portability and Accountability Act (HIPAA).

\subsection{Diffusion metrics}

We investigated direct biomarkers of trauma across the corpus callosum, thalamus, and hippocampus. These regions of interest were selected because they are deep brain structures that typically show changes after mild TBI (Niogi and Mukherjee 2010; Shenton et al. 2012; Eierud et al. 2014). We focused on the calculation of fractional anisotropy (FA) and increased mean diffusivity (MD) because concurrent observation of decreased FA and increased MD is a clinical marker of long-term injury. The diffusion data were preprocessed and corrected for motion and eddy currents using Eddy tool (FSL 5.0, FMRIB, Oxford University) (Andersson and Sotiropoulos 2016) and distortion using a field map. Fractional anisotropy (FA) and mean diffusivity (MD) maps were computed using the standard tensor diffusion model (dtifit, FSL). To segment the corpus callosum, thalamus, and hippocampus into distinct anatomical segments, we employed the FreeSurfer 5.3 pipeline (Dale et al. 1999), which uses the whole-brain structural T1-weighted image to parcellate the corpus callosum into five segments (anterior, mid-anterior, center, mid-posterior and posterior), the thalamus into two segments (left and right), and the hippocampus into two segments (left and right). Voxels at the edges were removed from the segmentations to account for partial voluming effects. We registered the diffusion $b 0$ T2-weighted images to their respective T1-weighted scans in FreeSurfer space using an affine transform and a normalized mutual information similarity metric (Flirt, FSL) (Jenkinson et al. 2002), manually inspecting each registration to confirm accurate alignment. Diffusion maps (FA and MD) were then transformed to the FreeSurfer space using the resulting registration, and the diffusion metrics computed along the five segments of the corpus callosum, the two segments of the thalamus and the two segments of the hippocampus for all players. FA/MD were normalized with respect to the average FA/MD value in the whole white matter (WM) to account for diffusion changes due to scanner drift or hardware changes across different time points (Baur et al. 2013).

\subsection{Statistical analysis}

Pearson's correlation coefficients $(r)$ were computed between peak components of translational and rotational acceleration from instrumented mouthguards, falx nodal displacements from FE simulations, and brain structure strains from FE simulation, with 1 indicating the strongest possible correlation and 0 indicating none. To compute confidence intervals around sample means, we used bootstrapping with 10,000 samples and the bias-corrected and accelerated percentile method to avoid assuming an underlying distribution. For each of the 115 measured head impacts, we extracted peak acceleration in six degrees of freedom. We used a nonparametric Wilcoxon rank-sum test to test whether combinations of these samples came from distributions with equal medians (without assuming anything about their underlying distribution) at a 5\% significance level.

\section{Results}

\subsection{Head acceleration measurements}

We analyzed previously published head kinematics of 115 sports impacts ( $2 \mathrm{mTBI}$ ) measured with instrumented mouthguards (Hernandez et al. 2015a). The biggest differences between injury impacts and sub-concussive peak head accelerations were found in coronal rotation, horizontal rotation, and left-right translation (Fig. 5). For coronal acceleration, the AOC mTBI had a peak of $12,900 \mathrm{rad} / \mathrm{s}^{2}$ while the SR mTBI had a peak of $4294 \mathrm{rad} / \mathrm{s}^{2}$. Sub-concussive mean (and confidence interval) was $1412 \mathrm{rad} / \mathrm{s}^{2}(1205-1674 \mathrm{rad} /$ $\mathrm{s}^{2}$ ) (Fig. 5). For horizontal rotation, the AOC mTBI had a peak of $3243 \mathrm{rad} / \mathrm{s}^{2}$ while the SR mTBI had a peak of $1983 \mathrm{rad} / \mathrm{s}^{2}$. Sub-concussive mean (and confidence interval) was $1049 \mathrm{rad} / \mathrm{s}^{2}\left(911-1228 \mathrm{rad} / \mathrm{s}^{2}\right)$ (Fig. 5). In contrast, AOC and SR mTBI impacts had sagittal rotational accelerations of $3057 \mathrm{rad} / \mathrm{s}^{2}$ and $5908 \mathrm{rad} / \mathrm{s}^{2}$, respectively, which were also typical for the sub-concussive group, with the mean of $3254 \mathrm{rad} / \mathrm{s}^{2}\left(2752-3868 \mathrm{rad} / \mathrm{s}^{2}\right)$ (Fig. 5). Coronal acceleration in the AOC mTBI $\left(12,900 \mathrm{rad} / \mathrm{s}^{2}\right)$ was nearly 3 standard deviations above the non-injurious mean and showed the largest difference than in any other rotation or translation direction. On the same day of injury, the SR injury subject experienced other 5 head impacts that did not exceed the injury impact in all accelerations except superior translation (Fig. 5). With the Wilcoxon rank-sum test, we rejected the hypothesis that any pair of the $6 \mathrm{DOF}$ accelerations comes from distributions with equal medians except for coronal and horizontal rotational acceleration at a 5\% significance level. 


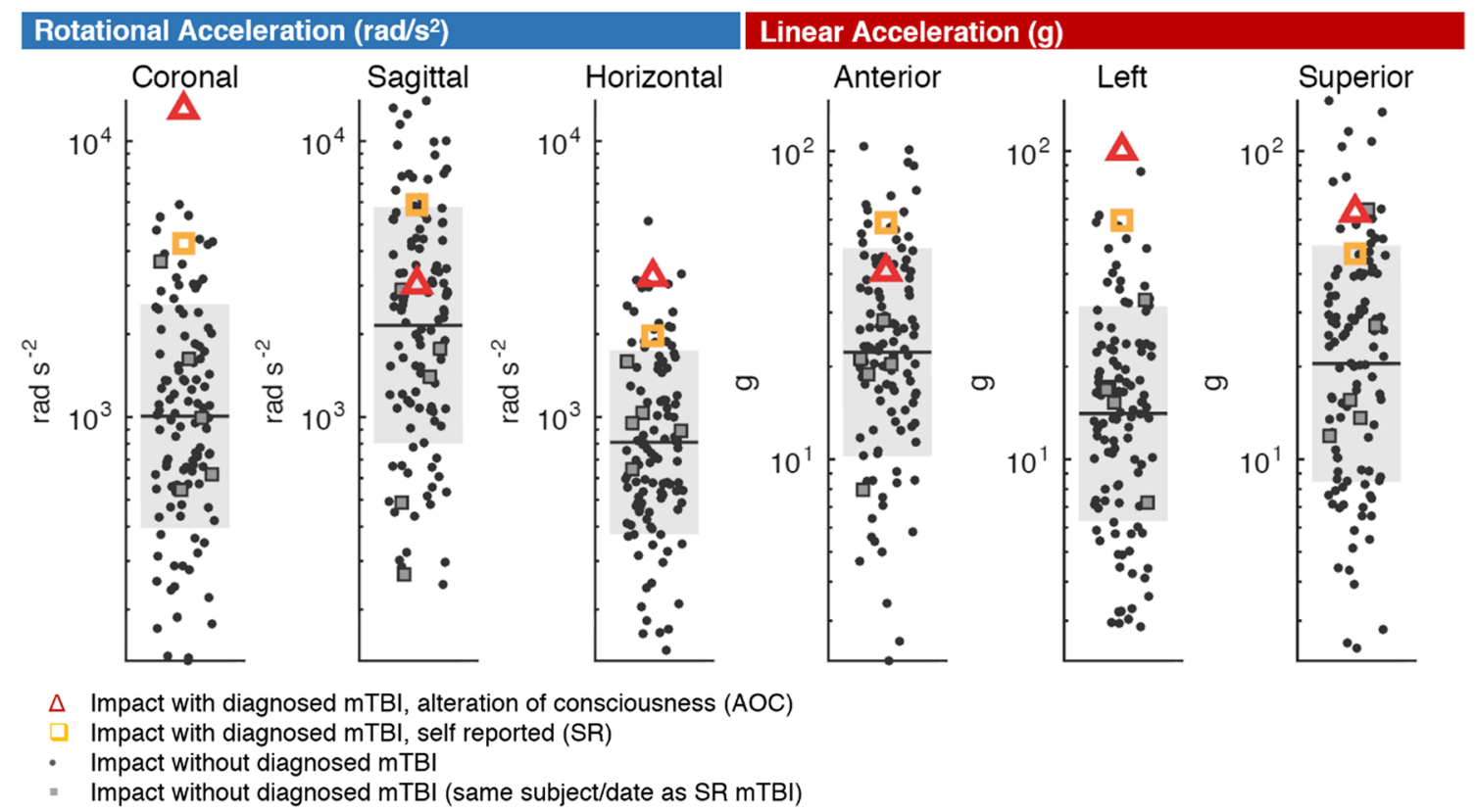

Fig. 5 Six-degree-of-freedom accelerations in mTBI impacts. Rotational accelerations in coronal, sagittal, and horizontal planes are plotted for 115 measured impacts, including a diagnosed injury that resulted in alteration of consciousness (AOC) and another that was self-reported (SR). Translational accelerations in anterior, left, and

This means that peak accelerations in these rotation planes followed similar distributions (supplementary material S2).

\subsection{Motion of the falx cerebri}

Some acceleration components were strongly correlated with deep (proximal) motion of the falx cerebri. Peak displacement of the falx cerebri was more than an order of magnitude larger in the left-right (lateral) direction than in the anterior-posterior and superior-inferior directions (Fig. 6). Peak lateral displacement of the falx anterior and posterior segments correlated strongly $(r>0.78)$ with peak horizontal rotational acceleration (Fig. 7a, c). Lateral displacement of the falx at its center correlated strongly $(r=0.85)$ with peak coronal acceleration (Fig. 7b). Lateral displacement at the center of the falx was also correlated with left translational acceleration $(r=0.75)$. However, lateral displacement of the falx cerebri was reproduced on average across the nodes at $56 \%$ and $77 \%$ by simulating the coronal and horizontal components alone, respectively (Fig. 7e); translation components produced minimal falx displacement (Fig. 7d, e). In the lateral direction, simulations with only the coronal component produced larger displacements of the deep falx center, whereas horizontal rotation produced larger motion in the periphery (anterior and posterior aspects) of the falx (Fig. 7d, e). Since these rotation directions were largest among injured subjects, it would follow, then, that superior planes are also provided. The impact producing the AOC injury is marked by unusually high coronal rotation and left translation where it is nearly 3 standard deviations above the non-injury median

the injured subjects experienced the largest lateral falx displacements.

The biggest mTBI distances from non-injurious mean were found for falx lateral displacement and speed (Fig. 6). The average lateral displacement of the 5 deep falx nodes (proximal to the center of gravity of the head) was about 200 times larger than that of the 6 peripheral nodes, close to the falx attachment to the skull. For deep lateral falx displacement, mTBI impacts experienced a mean displacement across all the proximal nodes of $6.0 \mathrm{~mm}(4.5-7.5 \mathrm{~mm}) \mathrm{com}-$ pared to $2.3 \mathrm{~mm}(2.1-2.5 \mathrm{~mm})$ for sub-concussive impacts (Fig. 8). The AOC mTBI impact produced the largest lateral falx displacement $(7.5 \mathrm{~mm})$ among all impacts, and it occurred in the centermost deep node. In this impact, peak lateral displacement of the falx was to the left and occurred at time $t=0.028 \mathrm{~s}$. This motion was coherent with the player's head acceleration occurring in the coronal plane counterclockwise (supplementary material S3). The SR mTBI also had its peak lateral displacement $(4.5 \mathrm{~mm})$ at the centermost deep node. Similar to the AOC injury, the left displacement peak coincided with counterclockwise coronal rotation (to the left) of the head. Both the mTBI impacts, which were characterized by high coronal acceleration, produced ' $\mathrm{C}$ '-shaped peak falx displacements in axial view. Sub-concussive impacts spanned a smaller range of lateral displacements. Also, their peaks tended to not occur in the centermost falx nodes, but rather, in more peripheral nodes, 


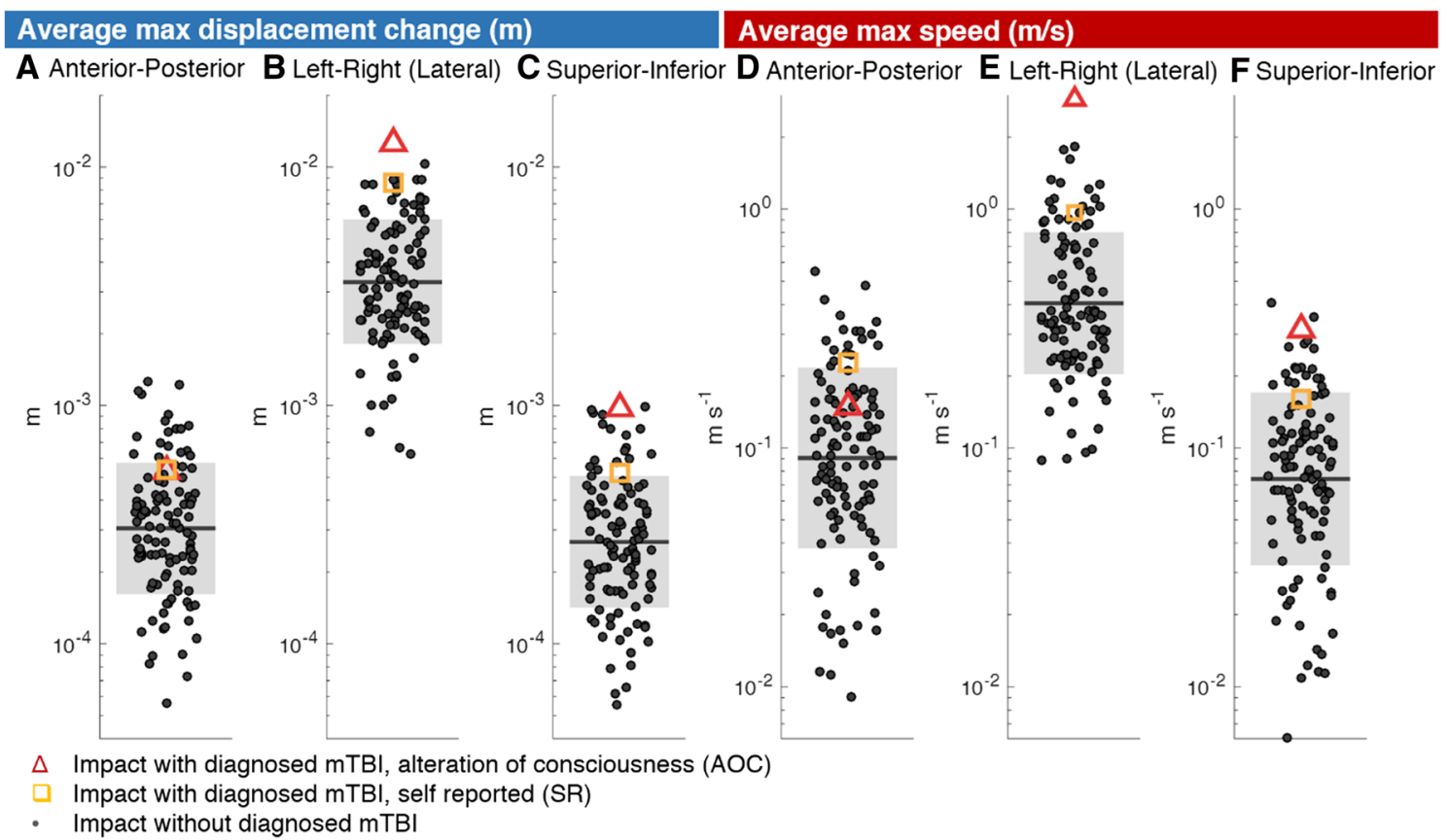

Fig. 6 Simulated falx cerebri displacement and speed in measured head impacts. Change in displacement of the falx cerebri in the a anterior-posterior, b left-right (lateral), and $\mathbf{c}$ superior-inferior directions are plotted for 115 measured impacts, including a diagnosed injury that resulted in alteration of consciousness (AOC) and another

drawing a 'S'-shape (Fig. 8). This was particularly true for impact characterized by high horizontal acceleration.

\subsection{Corpus callosum strain}

Simulated falx displacement and measured head kinematics were correlated with simulated white matter tract-oriented strain in different brain structures. Peak overall tract strain in the corpus callosum had strong correlation with lateral displacement of the falx center $(r=0.82)$ and coronal rotational acceleration $(r=0.80)$ (Fig. 9a). We found, however, that this relationship varied for substructures within the corpus callosum. While tract strain in the corpus callosum body correlated strongly with lateral displacement of the falx center $(r=0.91)$ and coronal rotation $(r=0.86)$, strain in the genu correlated more with peripheral lateral falx displacement (posterior $r=0.75$ ). For the entire corpus callosum and its substructures, sagittal rotation had poor correlation with tract strain $(r<0.63)$. In contrast, for all other brain structures, sagittal rotation had a strong correlation with tract strain $(r>0.88)$ (Fig. 9b). However, the magnitude of the tract strain produced by sagittal rotation was an order of magnitude smaller than the tract strain produced by coronal and horizontal rotation (regression coefficient $1.70-4.53$ vs. 3.17-12.72). Additionally, as shown in Fig. 9c, rotational acceleration in the horizontal plane produced higher tract that was self-reported (SR). Speed of the falx cerebri in the $\mathbf{d}$ anterior-posterior, e left-right (lateral), and $\mathbf{f}$ superior-inferior directions is also provided. The impact producing the AOC injury is marked by unusually high lateral displacement and speed where it is over 2 standard deviations above the non-injury median (gray box)

strain in all regions of the corpus callosum than in the coronal and sagittal planes. Peak overall tract strain in the thalamus also showed some correlation with lateral displacement of the falx center $(r=0.75)$ and coronal rotational acceleration $(r=0.75)$ (Fig. 9a). However, this correlation was weaker with respect to the corpus callosum ( $r=0.75$ vs. $r=0.82$ ).

We investigated a possibly causal relationship between falx motion and corpus callosum strain by running computer simulations of the AOC injury with geometry and material property modifications to the original FE model. Peak white matter tract-oriented strain in the corpus callosum for the original (normal brain) model was $35 \%$. We simulated the AOC injury head accelerations for different falx stiffnesses (changes in Young's modulus) and found that stiffening and softening the falx increased $(41 \%)$ and decreased (32\%) corpus callosum strain, respectively (Fig. 10). When the falx, dura, and pia mater were removed altogether, leaving only the longitudinal fissure with cerebrospinal fluid inside, corpus callosum strain was halved to $17 \%$, while other brain structures were mostly unaffected. Removing the fissure by filling it in with brain tissue did not produce any additional decrease in corpus callosum strain. However, it produced much larger strains in the cerebrum along the periphery. Finally, we tested model modifications that did not involve the falx, and found that adding a stiff or soft 

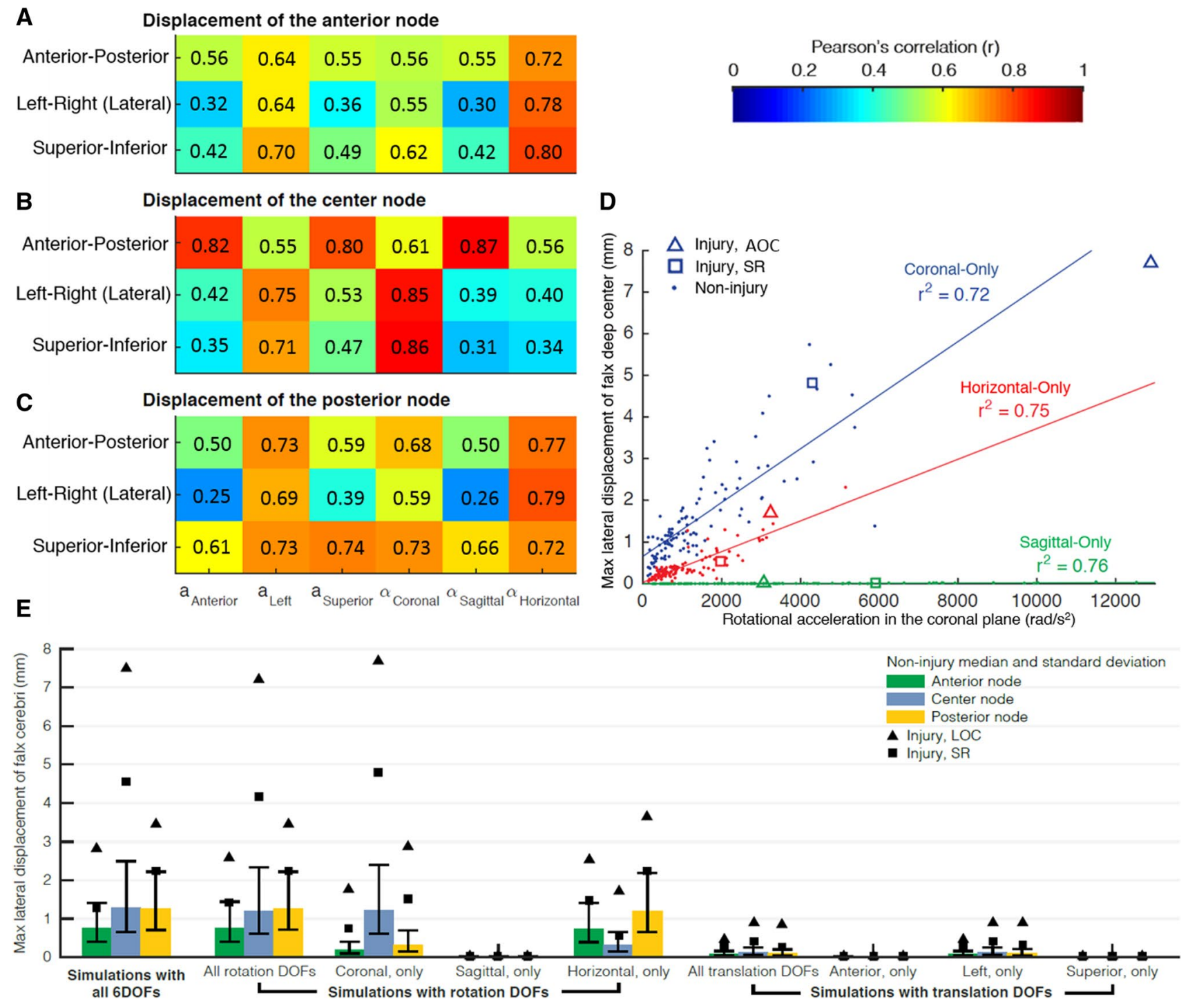

Fig. 7 Relationship between simulated displacement of the falx cerebri and measured head accelerations. Pearson's correlation coefficient $(r)$ relates peak head acceleration components with max displacement of anterior (a), central (b), and posterior (c) falx cerebri nodes. Coronal rotation is most strongly correlated with lateral and superior-inferior motion of the central portion of the falx $(r>0.85)$.

septum pellucidum did not substantially affect tract strain in any structure. Removing the ventricles by filling them in with brain tissue similarly did not affect tract strain in any structure (Fig. 10). The falx cerebri thus had a unique relationship with the peak tract strain in the corpus callosum (Fig. 10, red line).

We also investigated whether corpus callosum strain and strain rate distinguished the mTBI impacts. As it can be seen in Fig. 11, tract-oriented strain, strain rate and strain $\times$ strain rate appeared to be higher in the corpus callosum for the AOC and SR injuries compared to the median of non-injury impacts. Seven non-injurious impacts exceeded the mTBI
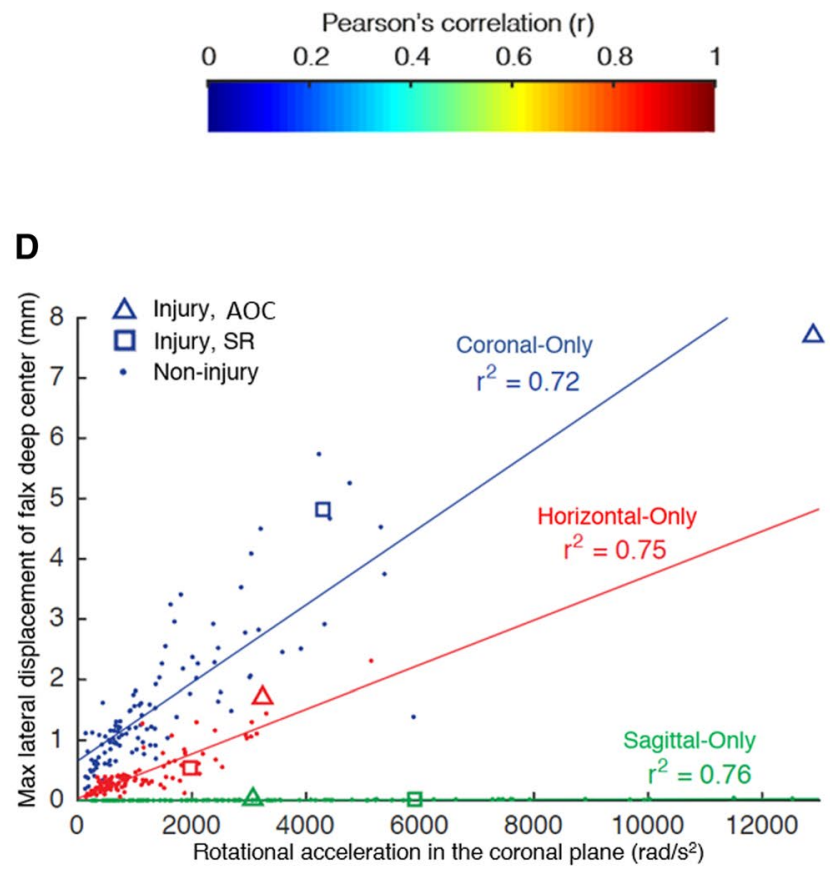

d Rotational acceleration in the coronal plane produces more lateral displacement in the falx deep center than in the horizontal and sagittal planes. e Peak displacement of the falx cerebri was more than an order of magnitude larger in the left-right (lateral) direction than in the anterior-posterior and superior-inferior directions and was mostly generated by rotation level $(6.1 \%)$ in tract-oriented strain. Six non-injurious impacts exceeded the mTBI level $(5.2 \%)$ in a combination of peak tract-oriented strain and strain rate. For this very small dataset, tract-oriented strain rate seemed to separate injurious from non-injurious cases best with five non-injurious impacts exceeding the mTBI level (4.3\%). Differences among the predictors were, however, minimal.

\subsection{DTI region of interest analysis}

Parallel to our modeling efforts, we looked for evidence of corpus callosum trauma in diffusion tensor imaging of the 


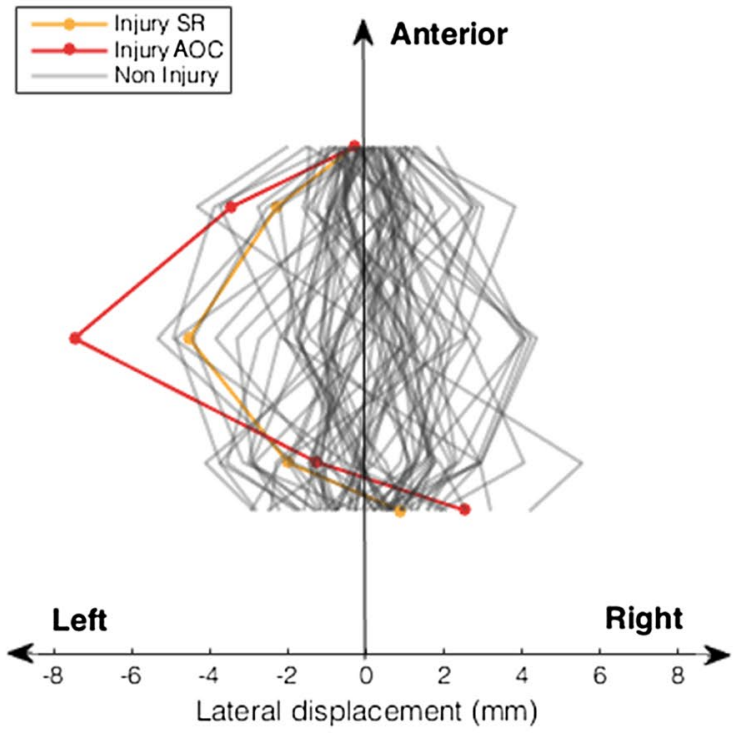

Fig. 8 Deep lateral displacement of the falx cerebri from FE simulations. Lateral displacement is depicted for 11 nodes on the falx cerebri: 6 distal (superior) and 5 proximal (inferior). The AOC injury had the largest lateral simulated falx displacement $(7.5 \mathrm{~mm})$ among

injured football players whose kinematics were simulated using FE models. We similarly investigated thalamus and hippocampus, which can have post-traumatic changes in mTBI. We observed some differences in deep brain structure imaging of the alteration of consciousness and self-reported injury compared to football players with no prior history of mTBI but from the same team with a similar number of years of experience in playing football. Figure 12 represents the normalized value of fractional anisotropy (FA) of the two concussed football athletes and control football players across the regions of interest. Our results showed lower FA values for the AOC injury than control football players in the anterior and mid-posterior segments of the corpus callosum. One day post-injury, the concussed athlete with reported symptoms (SR injury) showed FA values larger than the control median +1 SD in the mid-anterior and central segment of the corpus callosum (normalized FA $=1.47$ and 1.52 , respectively). We observed a subsequent decrease in FA values in the follow-up scan in the SR injury consistently across all corpus callosum segments, with the biggest change in the posterior segment $(-0.14)$ followed by the anterior segment of the corpus callosum $(-0.11)$. The SR injuries follow-up FA values, however, did not drop to the level of the follow-up scan of the AOC injury in all segments but the anterior. FA values in the thalami and hippocampi were instead close to control median or within $1 \mathrm{SD}$ and did not exhibit particular reduction trends for both the injurious cases.

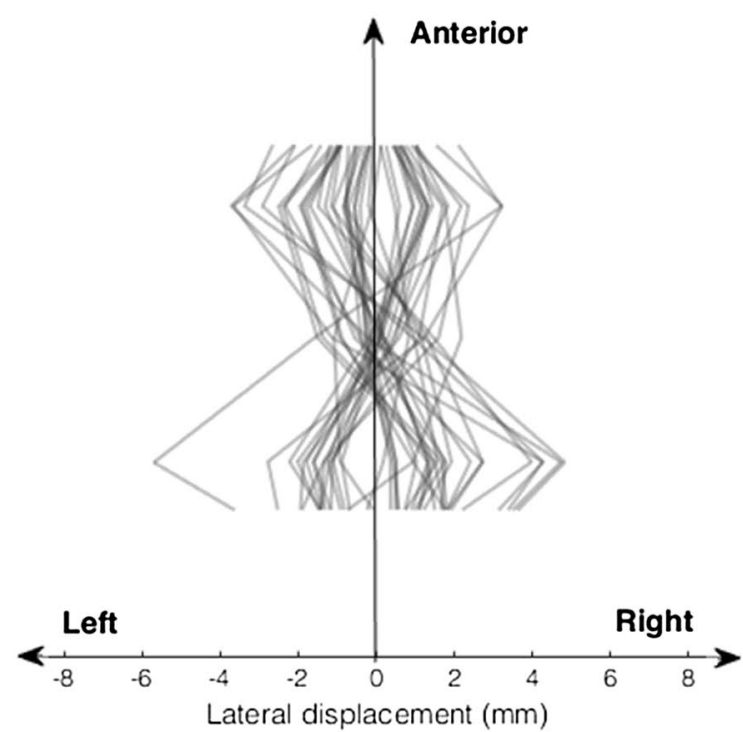

all impacts. The SR injury also had its peak simulated lateral displacement $(4.5 \mathrm{~mm})$ in the centermost deep node. Non-injury impacts spanned a smaller range of simulated lateral displacement, with peaks occurring in nodes offset from the center

We found an expected inverse trend for the normalized value of mean diffusivity (MD) (Fig. 13). The follow-up scan of the AOC injury exhibited increased MD (1.25) compared to the control median in the mid-posterior segment of the corpus callosum. This was in agreement with the biggest abnormality in FA value that was also located in the midposterior segment of the corpus callosum. For the AOC injury, we also found increased MD values in the right and left thalami, while MD values in the hippocampi were very close to control median. For the SR injury, MD increased in the follow-up scan, and the biggest change occurred in the posterior segment of the corpus callosum $(+0.13)$ followed by the left segment of the hippocampus $(+0.12)$ and the mid-posterior segment of the corpus callosum $(+0.09)$. The SR injuries follow-up MD values, however, did not increase to the level of the follow-up scan of AOC injury, except in the anterior segment of the corpus callosum and right thalamus.

\section{Discussion}

Trauma to the corpus callosum has been long implicated in mTBI studies, in humans and animals, in studies spanning histopathology to neuroimaging. Yet, the mechanism by which deformation penetrates this deep brain structure is unknown. While prior work implicated skull rotation and corpus callosum strain in mTBI, it was unclear if and how 


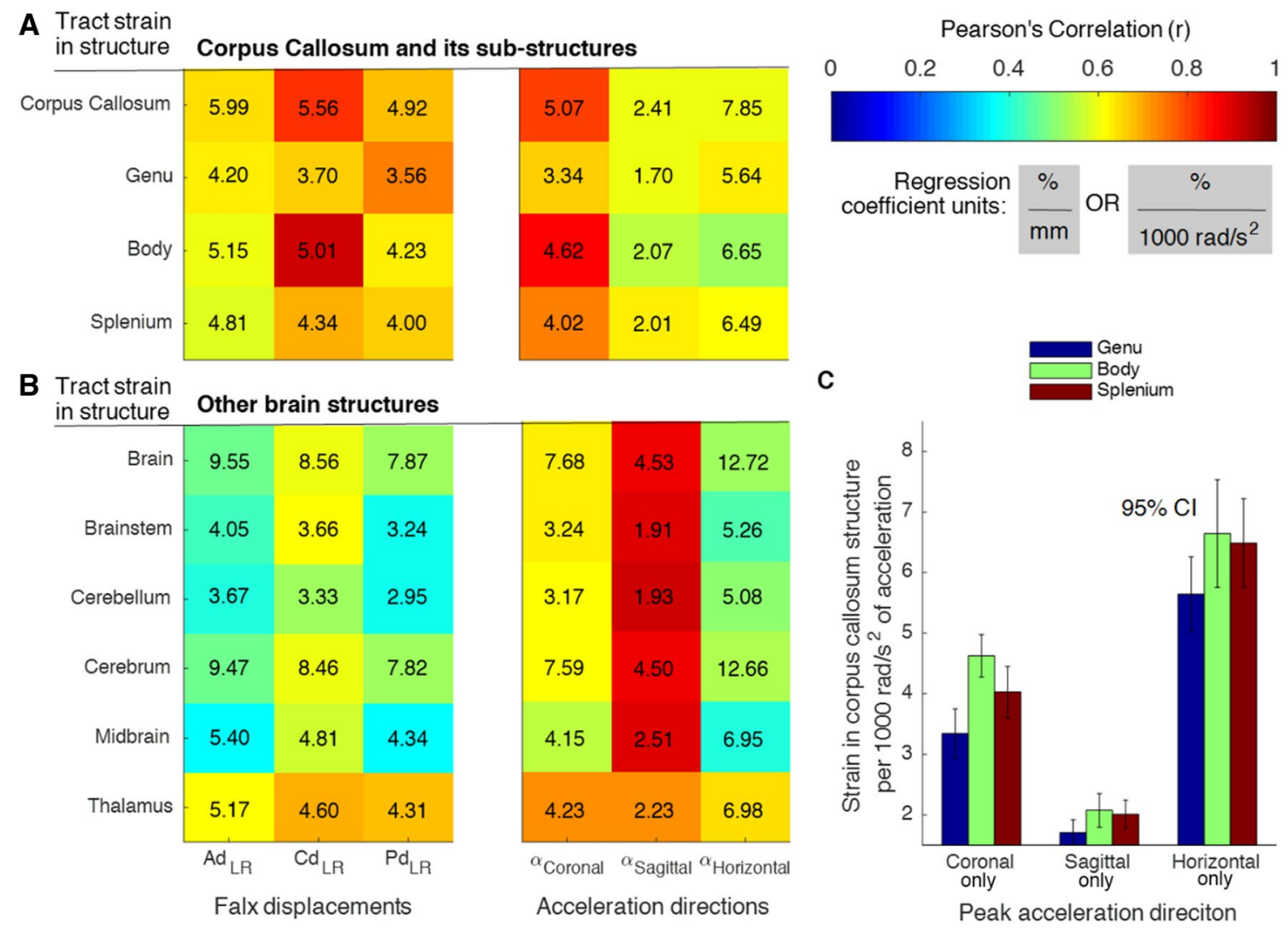

Fig. 9 Correlations with corpus callosum and other brain strains from FE simulations. For all impacts, Pearson's correlation $(r)$ and the linear regression coefficient relate simulated falx displacements and measured head accelerations to simulated peak tract strains in a the corpus callosum and $\mathbf{b}$ other brain structures. $\mathrm{Ad}, \mathrm{Cd}$, and $\mathrm{Pd}$ stand for anterior corpus callosum displacement, central corpus callosum displacement, and posterior corpus callosum displacement, respectively. c Rotational acceleration in the horizontal plane produced more tract strain in all regions of the corpus callosum than in the coronal and sagittal planes in FE simulations

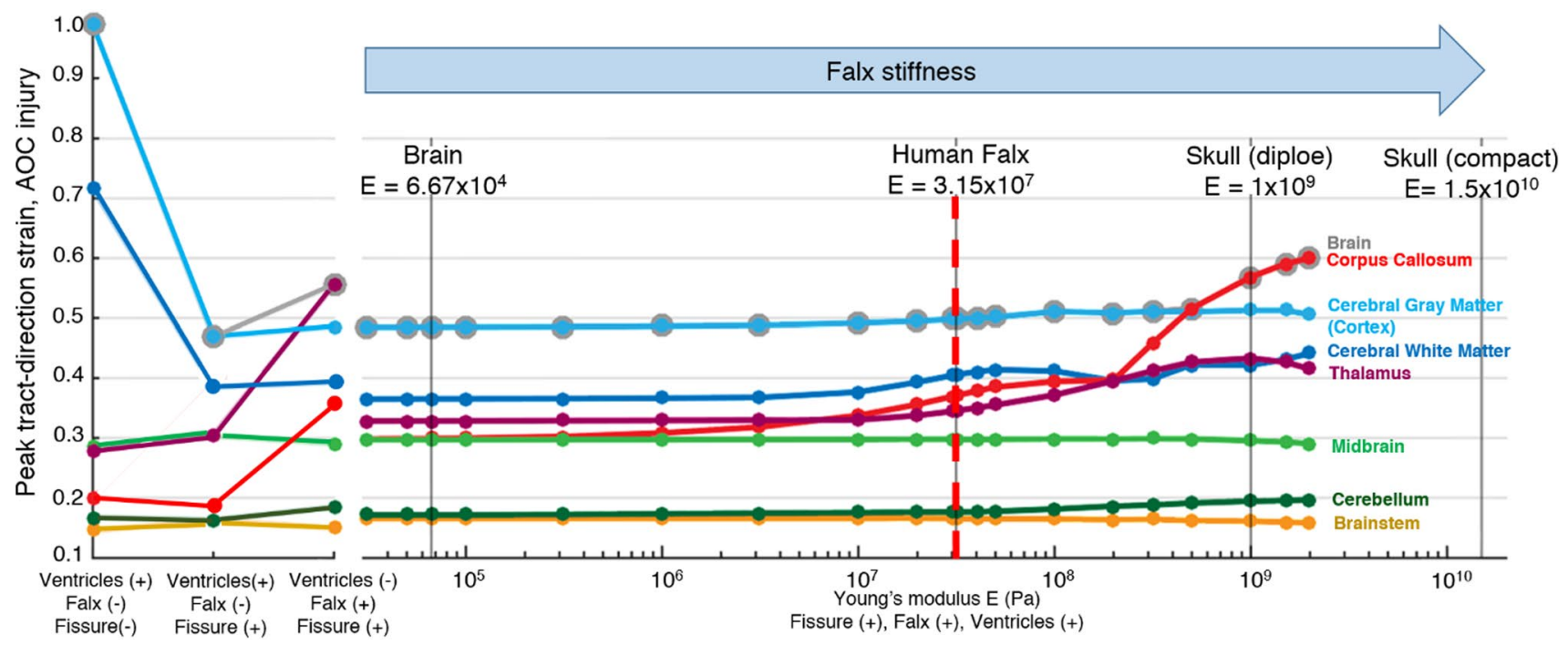

Fig. 10 The unique relationship of falx cerebri and corpus callosum tract strain. Computer simulations of the AOC injury with varying falx geometry and material properties found that removing the lon- gitudinal fissure and reducing falx stiffness reduced corpus callosum tract strain. The falx cerebri had a unique effect on the strain in the corpus callosum (red line) 

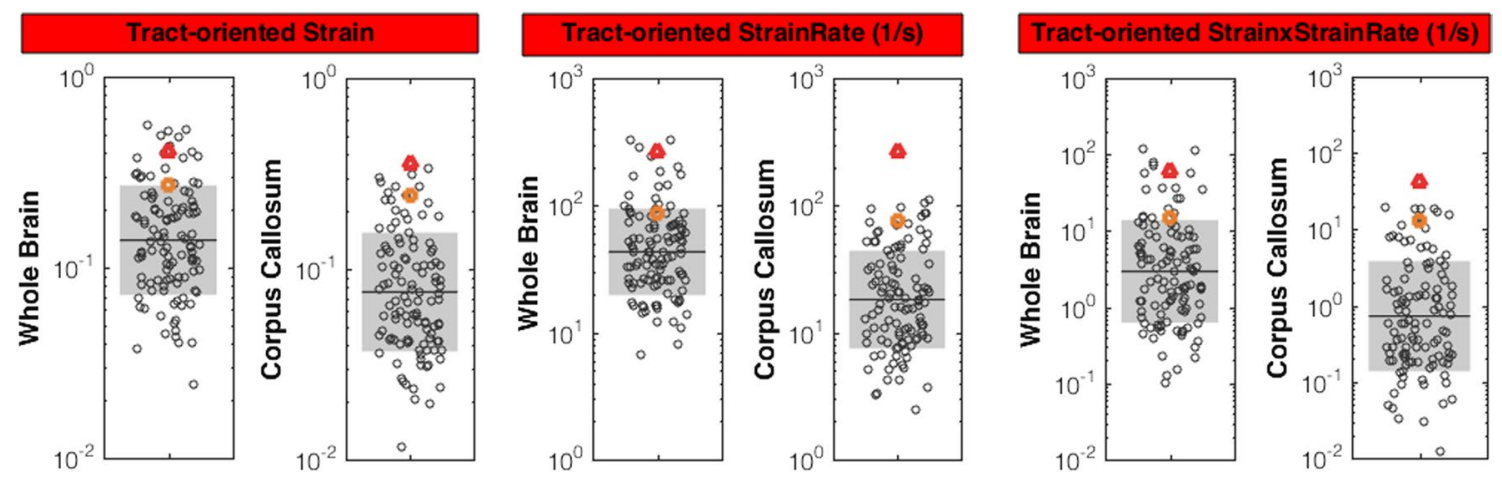

$\triangle$ Impact with diagnosed mTBI, alteration of consciousness (AOC)

$\square$ Impact with diagnosed $\mathrm{mTBI}$, self reported (SR)

- Impact without diagnosed mTBI

Fig. 11 Clinical relevance of brain tract strain and strain rate. Peak tract-oriented strain, strain rate $(1 / \mathrm{s})$, and strain $\times$ strain rate $(1 / \mathrm{s})$ are plotted for 115 measured impacts, including a diagnosed injury that resulted in alteration of consciousness (AOC) and another that was self-reported (SR)

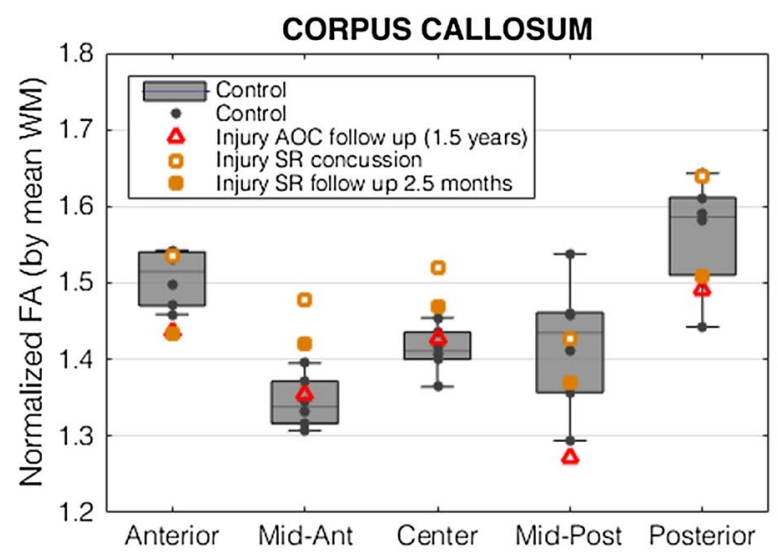

Fig. 12 Normalized fractional anisotropy from concussion and follow-up DTI scans. Diffusion tensor imaging was performed for the AOC mTBI subject a year and a half after his injury. For the SR mTBI, we had an acute DTI scan and a follow-up after 2.5 months from injury. The control was composed of 6 healthy football players. Compared to the average control subjects, the AOC mTBI subject appears different along the mid-posterior tract of the corpus callosum these were related. In this study, we explored the relationship between head motion, falx displacement, and corpus callosum trauma. We suggested a possible mechanism of corpus callosum trauma in sport-related concussion, and we looked for evidence of this trauma in diffusion tensor imaging of two concussed collegiate football players. Our results suggest that the corpus callosum may be sensitive to lateral impacts that cause coronal and horizontal rotations because they drive lateral motion of a relatively stiff membrane, the falx, in the direction of commissural fibers below. From modeling and imaging, we also found possible involvement of the thalami following coronal and horizontal rotation. However, this dataset is very limited because obtaining MRI and mouthguard data prospectively in matched cohorts is very challenging, but this study shows the promise of this novel approach. Future studies with a larger sample size combining baseline, longitudinal, and post-concussion DTI scans with kinematics data from instrumented mouthguard with DTI MRI findings will further explore these effects on deep white matter axons.

We studied the mechanism of putative corpus callosum injury, which we define as excessive strain in the corpus callosum and the symptoms that follow as a result. Using sixdegree-of-freedom (6DOF) head acceleration datasets, we found that peak coronal and horizontal rotational acceleration showed the biggest differences between mTBI and mean of sub-concussive head impacts. In finite element simulations, sagittal rotations caused relatively small displacement 


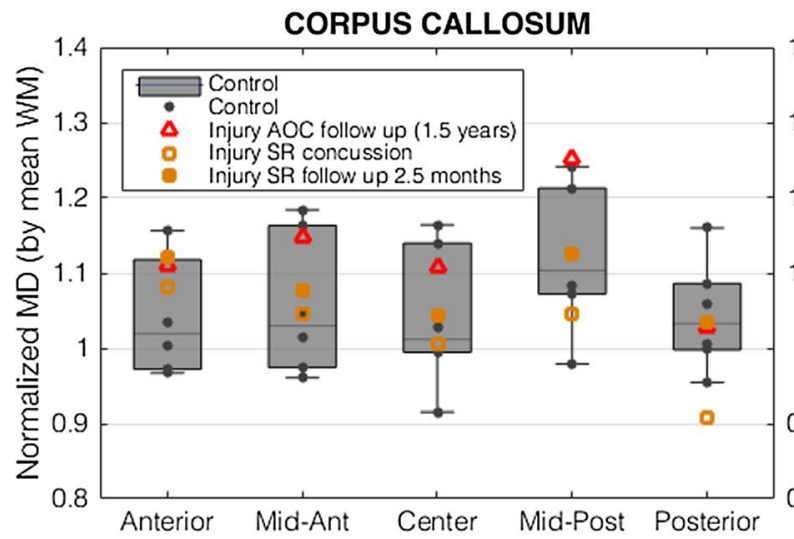

Fig. 13 Normalized mean diffusivity from concussion and followup DTI scans. Diffusion tensor imaging was performed for the AOC mTBI subject a year and a half after his injury. For the SR mTBI, we had an acute DTI scan and a follow-up after 2.5 months from injury.

of the falx strain on the corpus callosum. In contrast, coronal rotation was correlated with deep lateral displacement of the center of the falx cerebri $(r=0.85)$, while horizontal rotation was correlated with peripheral lateral motion of the falx cerebri $(r>0.78)$ (Fig. 7). We found relatively minor motion of the falx in the anterior-posterior (A/P) and inferior-superior $(\mathrm{I} / \mathrm{S})$ directions, with the peak falx displacement being more than an order of magnitude larger in the lateral direction than A/P and I/S (cm vs mm, Fig. 6). Furthermore, the mTBI cases showed C-shaped peak falx displacement mode (in axial view) while sub-concussive impacts spanned a smaller range of lateral displacements, conforming to an S-shape mode (Fig. 8). Peak left translational acceleration also appeared to be different for the injury group; this was expected given this direction of translation was correlated with coronal head rotation. In general, impact directions were not uncoupled. It is possible that complex combination of coronal and horizontal accelerations may have produced different mechanisms of injury: for example, horizontal rotation may have actually magnified (or softened) the traumatic effects of coronal rotation. However, we treated acceleration directions as independent, which was consistent with what done in Gennarelli et al. (1982, 1987); Smith et al. (2000); Browne et al. (2011); Eucker et al. (2011). Interestingly, our finding was in agreement with primate inertial injury studies that found isolated coronal rotations produced the most severe cases of diffuse axonal injury, followed by horizontal and then sagittal (Gennarelli et al. 1982, 1987).

From FE simulations, we investigated the correlation between lateral displacement of the falx and white matter tract-oriented strain in several brain structures. Several studies indeed showed that white matter tract-oriented strain is a mechanistically and clinically relevant predictor of mTBI (Giordano and Kleiven 2014; Ji et al. 2015). We found that lateral displacement of the central portion of the falx was most correlated with tract-oriented strain in the corpus callosum $(r=0.82)$. A correlation was found also with the thalamic region of the brain $(r=0.75)$ and may be due to the proximity of the thalamus to the corpus callosum. The thalamic region indeed seemed to be affected to some extent, suggesting a possible relationship between corpus callosum and thalamus deformation or at least an involvement of the thalamic region.

Horizontal rotation seemed to affect the corpus callosum as well. Although horizontal rotation was less correlated with the tract strain $(r<0.64)$, we found that it produced more strain in all regions of the corpus callosum (when normalized by angular acceleration) than any other degree of freedom (Fig. 9c). In other computational simulations of the human brain (Weaver et al. 2012), horizontal rotation was already shown to produce the largest strains in the brain overall. This also agrees with the brain injury criterion (BrIC), which is being considered for adoption by the National Highway Traffic Safety Administration (NHTSA) for New Car Assessment Program (NCAP) ratings (Takhounts et al. 2008). BrIC weighs horizontal rotation as the least tolerable direction while coronal rotation is weighed as the most tolerable, opposite of maximum head velocities humans are capable of producing voluntarily (Hernandez and Camarillo 2018) and likely because overall brain strain was considered when creating the criteria. Determining direction dependence to overall brain strain may be not sufficient, as deformation patterns are complex and rotation planes may correlate with trauma to specific brain structures, as with coronal plane rotation and the corpus callosum in the present study.

Our hypothesis is that high coronal rotation and/or horizontal rotations produce deep lateral displacement of the 
falx cerebri, which in turn may strain and damage the corpus callosum. To develop this hypothesis, we ran computer simulation to investigate the relationship between falx motion and corpus callosum strain. We selected the kinematics of the AOC injury and varied material properties and geometry of the original FE model. Interestingly, we found that increasing the Young's modulus of the falx (stiffening the falx) increased the tract strain in the corpus callosum. This relationship was marked and unique between the falx and the corpus callosum (Fig. 10, red line). Because removing the falx and leaving the inter-hemispheric fissure halved corpus callosum strain, our simulations do not support the hypothesis that the presence of the brain fissure alone causes injury through a stress concentration. Our finding is in agreement with other studies using $2 \mathrm{D}$ or $3 \mathrm{D}$ computational models (Nishimoto and Murakami 1998; Li et al. 2007; Ho et al. 2017), where it was found that the presence of the falx causes a steep stiffness gradient at the loose end of the membrane, which induces a strain concentration in the structures around it, namely the corpus callosum. However, physical model simulations of the baboon brain found the falx to be protective, reducing shear strains in the corpus callosum when present (Margulies et al. 1990). In these experiments, the falx was tethered to a Plexiglas cover plate that cut short the free (deep) edge of the falx and thus its available mobility. In our study, the falx was attached along the midline of the skull, which explains our observation of larger displacements distal to this attachment (proximal to the head center of gravity). Our study may contradict another hypothesis regarding falx motion; rather than constraining the corpus callosum by impinging motion of the trailing cerebral hemisphere (Smith et al. 2000), our results indicate that the falx may actually drive or lead the cerebral hemispheres just above the corpus callosum. Because it is much stiffer and reacts more quickly than brain tissue, the falx may have dynamics that are out of phase with motion of brain tissue whereby rapid direction changes could result in instances of large strain inferiorly.

To determine whether findings from our modeling efforts agreed with in vivo clinical observations, we looked for evidence of corpus callosum trauma in magnetic resonance imaging of the two injured football players whose kinematics were simulated using FE models. We investigated direct biomarkers of trauma in diffusion tensor imaging in regions of the brain typically damaged in mTBI, such as the corpus callosum, thalamus, and hippocampus. According to a review of over 10 years of DTI brain injury studies (Eierud et al. 2014), decreased FA is indeed frequently reported in the post-acute window following an mTBI ( $>2$ weeks) and correlates with neuropsychological performance. Moreover, increased MD is found in the chronic stage of injury and there is a negative correlation between MD and cognitive outcome measures such as attention, executive function, memory, psychomotor/processing speed, and visuospatial abilities (Niogi and Mukherjee 2010; Shenton et al. 2012; Eierud et al. 2014). Consequently, concurrent observation of decreased FA and increased MD is a clinical marker of long-term injury.

The subject experiencing the AOC mTBI was enrolled in the imaging study for follow-up only (1.5 years post-injury). As control, we used a population of six football players with no prior history of concussion. From DTI, we extracted normalized fractional anisotropy and mean diffusivity for anterior, mid-anterior, central, mid-posterior, and posterior segments of the corpus callosum, left and right thalami, and left and right hippocampi. After 1.5 years from the injury, the AOC mTBI subject showed fractional anisotropy $>3$ SD below the average control and increased mean diffusivity in the mid-posterior segment of the corpus callosum. This concurrent observation may indicate long-term injury in the corpus callosum, located at the interface between the body and the splenium. These imaging findings seem to be in agreement with our computational observations of large tract-oriented strain in the corpus callosum body: large deformation of the body may indeed have torn axons connecting the body and the splenium of the corpus callosum, permanently damaging the mid-posterior segment of the corpus callosum. However, given the point in time at which the scan was acquired and the lack of a same-subject baseline, caution needs to be exercised when interpreting results. Elevated values of MD were also found in the thalamus, which also had a tract strain correlation with the falx motion, but did not correspond to depressed FA values. This may indicate that deformation travelled from the falx to the corpus callosum and proceeded to the thalamus, producing damage in the thalamic area as well. The thalamus is a deep gray structure extensively connected to the neocortex, and in contradistinction to the tightly bundled corpus callosum, the thalamus contains more diffuse axonal fibers at more complex configurations. Potential injury to the thalamus may involve not only direct axonal damage but also direct cellular damage given the thalamus is a gray matter structure, as well as indirect damage from deafferentation of projecting white matter fibers. The finding of altered MD without alteration of FA may reflect an injury with primarily cellular damage. However, without baseline imaging of the injured athlete, future studies with a larger sample size, baseline imaging, and inclusion of multi-compartment diffusion models are necessary to confirm these effects on corpus callosum and thalamic axons and intra-axonal compartments.

We conducted DTI on the SR mTBI subject at 1 day post-injury (acute) and at 2.5 months post-injury (followup). For the SR mTBI subject, we observed a longitudinal decrease in FA values and increase in MD values in the follow-up scan from immediate post-injury scan particularly in the posterior segment of the corpus callosum. While the 
biggest longitudinal change of FA and MD was seen in the posterior segment of the corpus callosum $(-0.14$ and +0.13 , respectively), the diffusion metrics at follow-up were mostly within normal range. Depressed FA was found at the genu of the corpus callosum, while thalamus and hippocampus were not particularly affected. MD at follow-up was within normal range across all segments, except for the right thalamus. As for the AOC injury, elevated MD values in the thalamus did not correspond to depressed FA values and may be an index of an injury with primarily cellular damage. Also, reduced values of MD were found at acute stage in the left segment of the hippocampus and posterior segment of the corpus callosum, which is typically associated with the presence of acute cytotoxic edema. However, these values returned to the normal range at follow-up. A concurrent observation of depressed FA and elevated MD was found in the anterior segment of the corpus callosum, but was not as evident as in the AOC case. These DTI metrics are in line with our computational results, where falx lateral displacement was smaller for the SR injury than the AOC mTBI, but fell in the high non-injurious range (Fig. 8).

This study has several limitations to mention. The greatest limitations were the limited size of our injury cohort, which does not allow to perform logistic regression to estimate risk injury curves. To reduce the skewness of the dataset, we enriched the dataset for high acceleration noninjurious impacts that are the most difficult to distinguish from the mTBI cases. Along with both injuries, 50 non-injurious impacts were selected at random, 58 selected because they exceeded either injury in any translational or rotational acceleration component, and 5 selected because they were sustained by the self-reported mTBI subject on the same day (and prior to) his injury. The scope of this study is to propose a possible mechanism through which skull rotation generates deformation in the corpus callosum. The current dataset is too limited to infer injury thresholds or other injury metrics, and even more, to calculate confidence intervals. We divided the players in two binary groups - mTBI and subconcussive_-based on physician diagnosis. However, it may be possible that some axonal damage could be un-detected by imaging or symptom evaluation. We are currently collecting further data that will allow us to expand our dataset and estimate risk injury curves and confidence interval in future studies.

Another limitation was our dependence on finite element simulations to develop our theory of the corpus callosum injury and our explanation of the mechanism of trauma. The KTH finite element brain model was compared to displacement data from cadaver head impact experiments performed by (Hardy 2001) where neutral density targets were inserted inside cadaver brains and tracked using high-speed biplanar X-ray during impacts. On average, errors in $x$-, $y$-, and $z$-displacements for the KTH model were $1 \mathrm{~mm}$ or less for frontal, occipital, and lateral impacts (Kleiven 2006). Intracranial pressures from the cadaver experiments were also estimated by the FE model within 5\%. However, experiments on cadavers are only partially representative of the behavior of intact, living human brain. Moreover, measurements in the brain from cadavers are sparse. In the literature, data on human brain response in vivo are available from tagged MRI experiments (Bayly et al. 2005; Sabet et al. 2008; Feng et al. 2010). These living strain data have a much greater spatial resolution than the sparse displacement measurements from cadaver studies. To increase the confidence in predicting tissue strain in living subjects, we compared the model strain predictions on sub-injury strains measured using tagged MRI during frontal impact (supplementary material S1) (Feng et al. 2010). Although errors in predicting the maximum principal strain for the KTH model were $37 \%$ or less for an in vivo frontal impact (supplementary material S1), in these studies volunteers underwent deceleration of the head on the order of 2-3 g (100-300 rad/s $\mathrm{s}^{2}$ of angular acceleration). The average linear accelerations from the field were instead about $25 \mathrm{~g}$ for sub-concussive impacts and $>50 \mathrm{~g}$ for mTBI cases (1000-10,000 rad/s $\mathrm{s}^{2}$ of rotational acceleration) (Fig. 5), over an order of magnitude larger than in the experiments with volunteers. Because measurement of strains at injurious levels on living human brain is impossible, the direct assessment of the FE model under these conditions cannot be performed.

We used a relatively low mesh density FE model for the study, which limited the possibility of geometrical detail, especially when analyzing small regions of the corpus callosum. However, the volume of the brain structure did correlate well with MRI-based volumetric data (Kleiven 2007), and a comparison between the KTH model and high mesh density FE models showed a similar correlation with the cadaveric localized brain motion experiments (Kleiven 2007; Giordano and Kleiven 2014). In addition, the KTH FE model represents the 50th male percentile of the human head. When applying head impact kinematics from our participants to an average FE model, we approximated head geometry and material properties to the average of population. From the literature, it is known that uncertainties in the calculations may arise from variation in head size (Kleiven and Von Holst 2002; Ho and Kleiven 2009) and modeling choices (Ji et al. 2014). Study participants had, however, similar head dimensions and brain mass to the FE model on average (head length: $20.1 \mathrm{vs} 19.9 \mathrm{~cm}$; head width: $16.2 \mathrm{vs}$ $15.9 \mathrm{~cm}$; brain mass: $1.53 \mathrm{~kg}$ vs $1.42 \mathrm{~kg}$ ). Future work will involve more detailed FE models.

Finally, there were limitations in our imaging analysis. Imaging was performed on our AOC mTBI subject a year and a half after his injury. Baseline imaging pre-mTBI was not collected for the mTBI subjects, and consequently, it was impossible to know whether the observed differences were 
due to injury or individual differences between subjects and controls. However, our imaging control group consisted of football players with no previous history of concussion. Controls played football for a similar number of years and were recruited from the same football team as the mTBI cases. Because of age and head impact exposure-matching, controls should provide a quite reliable baseline. Nevertheless, we need to use caution when interpreting imaging results. In particular, head injury history may affect imaging interpretation. The AOC mTBI subject sustained another mTBI about 4 weeks prior to the studied mTBI. Long-term effects visible from the DTI may be due to a summation of events. Future studies with a larger sample size, baseline, and follow-up imaging are necessary to confirm current findings.

\section{Conclusion}

Our results found that sagittal rotation of the head has little effect on the corpus callosum. However, coronal and horizontal rotations caused lateral displacement of the falx cerebri at the center and periphery, respectively. These motions corresponded with strain in regions of the corpus callosum just below the location of falx displacement, which did not occur when the falx was removed. Supporting evidence from imaging indicated that the corpus callosum strain produced by some of our measured impacts may have been enough to yield tissue and clinical trauma. In addition, coronal and horizontal rotations seemed to affect the thalamic region to some extent. However, given the small size of our cohort of injury, more research is necessary to confirm our findings. Perhaps more importantly, our results illustrate that injury to different brain structures can be produced through different mechanisms, and require different detection criteria, prevention strategies, and/or treatments. By understanding mechanisms like those from this study, injury detection and prevention efforts can be focused on limiting specific high risk movements (e.g., a new helmet design to support the head in the coronal plane or redirect motion into another plane) (Hernandez et al. 2015b). Part of the novelty of this study is the combination of direct head rotation measurements with imaging findings. While collecting concurrent observations of injury is challenging, the method in this study can be applied to determine the mechanism of trauma to other brain structures and to confirm the results of the present investigation for non-computational datasets.

Acknowledgements We would like to acknowledge Dr. Svein Kleiven for providing the KTH brain model, which was used in this study to compute brain deformations and strains. We thank the Stanford Department of Athletics (Palo Alto, CA) for enabling this research. This work was supported by the Stanford Child Health Research Institute, Lucile Packard Foundation for Children's Health 38454, the National Institutes of Health (NIH) National Institute of Biomedical Imaging and
Bioengineering (NIBIB) 3R21EB01761101S1, Radiology Society of North America, and GE Healthcare.

\section{Compliance with ethical standards}

Conflict of interest The authors declare that they have no conflict of interest.

Open Access This article is distributed under the terms of the Creative Commons Attribution 4.0 International License (http://creativeco mmons.org/licenses/by/4.0/), which permits unrestricted use, distribution, and reproduction in any medium, provided you give appropriate credit to the original author(s) and the source, provide a link to the Creative Commons license, and indicate if changes were made.

\section{References}

Andersson JLR, Sotiropoulos SN (2016) An integrated approach to correction for off-resonance effects and subject movement in diffusion MR imaging. Neuroimage 125:1063-1078. https://doi. org/10.1016/j.neuroimage.2015.10.019

Arenth PM, Russell KC, Scanlon JM et al (2014) Corpus callosum integrity and neuropsychological performance after traumatic brain injury: a diffusion tensor imaging study. J Head Trauma Rehabil 29:1-16. https://doi.org/10.1097/HTR.0b013e318289ede 5

Arun KS, Huang TS, Blostein SD (1987) Least-squares fitting of two 3-D point sets. IEEE Trans Pattern Anal Mach Intell 9:698-700. https://doi.org/10.1109/TPAMI.1987.4767965

Bartsch A, Samorezov S, Benzel E et al (2014) Validation of an "intelligent mouthguard" single event head impact dosimeter. Stapp Car Crash J 58:1-27

Baur V, Brühl AB, Herwig U et al (2013) Evidence of frontotemporal structural hypoconnectivity in social anxiety disorder: a quantitative fiber tractography study. Hum Brain Mapp 34:437-446. https ://doi.org/10.1002/hbm.21447

Bayly PV, Cohen TS, Leister EP et al (2005) Deformation of the human brain induced. J Neurotrauma 22:845-856

Browne KD, Chen X-H, Meaney DF, Smith DH (2011) Mild traumatic brain injury and diffuse axonal injury in swine. J Neurotrauma 28:1747-1755. https://doi.org/10.1089/neu.2011.1913

Camarillo DB, Shull PB, Mattson J et al (2013) An instrumented mouthguard for measuring linear and angular head impact kinematics in american football. Ann Biomed Eng 41:1939-1949. https://doi.org/10.1007/s10439-013-0801-y

Cameron KL, Marshall SW, Sturdivant RX, Lincoln AE (2012) Trends in the incidence of physician-diagnosed mild traumatic brain injury among active duty U.S. Military Personnel between 1997 and 2007. J Neurotrauma 29:1313-1321. https://doi.org/10.1089/ neu. 2011.2168

Cassidy JD, Carroll LJ, Peloso PM et al (2004) Incidence, risk factors and prevention of mild traumatic brain injury: results of the WHO collaborating centre task force on mild traumatic brain injury. J Rehabil Med 36:28-60. https://doi.org/10.1080/1650196041 0023732

Coronado VG, McGuire LC, Sarmiento K et al (2012) Trends in traumatic brain injury in the U.S. and the public health response: 1995-2009. J Safety Res 43:299-307. https://doi.org/10.1016/j. jsr.2012.08.011 
Dale AM, Fischl B, Sereno MI (1999) Cortical surface-based analysis: I. Segmentation and surface reconstruction. Neuroimage 9:179194. https://doi.org/10.1006/nimg.1998.0395

Eierud C, Craddock RC, Fletcher S et al (2014) Neuroimaging after mild traumatic brain injury: review and meta-analysis. NeuroImage Clin 4:283-294. https://doi.org/10.1016/j.nicl.2013.12.009

Eucker SA, Smith C, Ralston J et al (2011) Physiological and histopathological responses following closed rotational head injury depend on direction of head motion. Exp Neurol 227:79-88. https ://doi.org/10.1016/j.expneurol.2010.09.015

Feng Y, Abney TM, Okamoto RJ et al (2010) Relative brain displacement and deformation during constrained mild frontal head impact. J R Soc Interface 7:1677-1688. https://doi.org/10.1098/ rsif. 2010.0210

Funk J, Cormier J, Bain C, et al (2009) Validation and application of a methodology to calculate head accelerations and neck loading in soccer ball impacts. SAE Int 2009-01-0251. https://doi. org/10.4271/2009-01-0251

Gazzaniga M, Irvy RB, Mangun GR (1988) Cognitive neuroscience the biology of the mind. W.W. Norton \& Company, New York

Gennarelli TA, Thibault LE, Adams JH et al (1982) Diffuse axonal injury and traumatic coma in the primate. Ann Neurol 12:564574. https://doi.org/10.1002/ana.410120611

Gennarelli T, Thibault L, Tomei G et al (1987) Directional dependence of axonal brain injury due to centroidal and non-centroidal acceleration. SAE Tech Pap Ser 872197:49-53. https://doi. org/10.4271/872197

Giordano C, Kleiven S (2014) Evaluation of axonal strain as a predictor for mild traumatic brain injuries using finite element modeling. Stapp Car Crash J 58:29-61

Giordano C, Cloots RJH, van Dommelen JAW, Kleiven S (2014) The influence of anisotropy on brain injury prediction. J Biomech 47:1052-1059. https://doi.org/10.1016/j.jbiomech.2013.12.036

Guskiewicz K, Mccrea M, Marshall SW et al (2003) The NCAA concussion study. J Am Med Assoc 290:2549-2555

Hardy W (2001) Investigation of head injury mechanisms using neutral density technology and high-speed biplanar X-ray. Stapp Car Crash J 45:337-368

Hardy WN, Mason MJ, Foster CD et al (2008) A study of the response of the human cadaver head to impact. Stapp Car Crash J 51:17-80

Hernandez F, Camarillo DB (2018) Voluntary head rotational velocity and implications for brain injury risk metrics. J Neurotrauma. https://doi.org/10.1089/neu.2016.4758

Hernandez F, Wu LC, Yip MC et al (2015a) Six degree-of-freedom measurements of human mild traumatic brain injury. Ann Biomed Eng 43:1918-1934. https://doi.org/10.1007/s10439-014-1212-4

Hernandez F, Shull PB, Camarillo DB (2015b) Evaluation of a laboratory model of human head impact biomechanics. J Biomech 48:3469-3477. https://doi.org/10.1016/j.jbiomech.2015.05.034

Ho J, Kleiven S (2009) Can sulci protect the brain from traumatic injury? J Biomech 42:2074-2080

Ho J, Zhou Z, Li X, Kleiven S (2017) The peculiar properties of the falx and tentorium in brain injury biomechanics. J Biomech 60:243-247. https://doi.org/10.1016/j.jbiomech.2017.06.023

Ivins BJ (2010) Hospitalization associated with traumatic brain injury in the active duty US Army: 2000-2006. NeuroRehabilitation 26:199-212. https://doi.org/10.3233/NRE-2010-0556

Jadischke R, Viano DC, Dau N et al (2013) On the accuracy of the head impact telemetry (hit) system used in football helmets. J Biomech 46:2310-2315. https://doi.org/10.1016/j.jbiomech.2013.05.030

Jenkinson M, Bannister P, Brady M, Smith S (2002) Improved optimization for the robust and accurate linear registration and motion correction of brain images. Neuroimage 17:825-841. https://doi. org/10.1016/S1053-8119(02)91132-8
Ji S, Ghadyani H, Bolander RP et al (2014) Parametric comparisons of intracranial mechanical responses from three validated finite element models of the human head. Ann Biomed Eng 42:11-24

Ji S, Zhao W, Ford JC et al (2015) Group-wise evaluation and comparison of white matter fiber strain and maximum principal strain in sports-related concussion. J Neurotrauma 32:441-454. https:// doi.org/10.1089/neu.2013.3268

Kleiven S (2006) Evaluation of head injury criteria using a finite element model validated against experiments on localized brain motion, intracerebral acceleration, and intracranial pressure. Int $\mathbf{J}$ Crashworthiness 11:65-79

Kleiven S (2007) Predictors for traumatic brain injuries evaluated through accident reconstructions. Stapp Car Crash J 51:81-114. https://doi.org/10.4271/2007-22-0003

Kleiven S, von Holst H (2002) Consequences of head size following trauma to the human head. J Biomech 35:153-160

Knox T (2004) Validation of earplug accelerometers as a means of measuring head motion. In: Proceedings of 2004 SAE motorsports engineering conference and exhibition 2004-01-3538

Kuo C, Wu LC, Hammoor BT et al (2016) Effect of the mandible on mouthguard measurements of head kinematics. J Biomech 49:1845-1853. https://doi.org/10.1016/j.jbiomech.2016.04.017

Li J, Zhang J, Yoganandan N et al (2007) Regional brain strains and role of falx in lateral impact-induced head rotational acceleration. Biomed Sci Instrum 43:24-29

Lincoln AE, Caswell SV, Almquist JL et al (2011) Trends in concussion incidence in high school sports: a prospective 11-year study. Am J Sports Med 39:958-963. https://doi. org/10.1177/0363546510392326

Margulies SS, Thibault LE, Gennarelli TA (1990) Physical model simulations of brain injury in the primate. J Biomech 23:823836. https://doi.org/10.1016/0021-9290(90)90029-3

Maroon JC, Winkelman R, Bost J et al (2015) Chronic traumatic encephalopathy in contact sports: a systematic review of all reported pathological cases. PLoS ONE 10:1-16. https://doi. org/10.1371/journal.pone.0117338

Montenigro PH, Alosco ML, Martin BM et al (2017) Cumulative head impact exposure predicts later-life depression, apathy, executive dysfunction, and cognitive impairment in former high school and college football players. J Neurotrauma 34:328-340. https://doi.org/10.1089/neu.2016.4413

Naunheim RS, Bayly PV, Standeven J et al (2003) Linear and angular head accelerations during heading of a soccer ball. Med Sci Sports Exerc 35:1406-1412. https://doi.org/10.1249/01. MSS.0000078933.84527.AE

Niogi SN, Mukherjee P (2010) Diffusion tensor imaging of mild traumatic brain injury. J Head Trauma Rehabil 25:241-255. https://doi.org/10.1097/HTR.0b013e3181e52c2a

Nishimoto T, Murakami S (1998) Relation between diffuse axonal injury and internal head structures on blunt impact. J Biomech Eng 120:140-147. https://doi.org/10.1115/1.2834294

Omalu BI, DeKosky ST, Minster RL et al (2005) Chronic traumatic encephalopathy in a National Football League player. Neurosurgery 57:128-133. https://doi.org/10.1227/01.NEU.0000163407 92769.ED

Omalu BI, DeKosky ST, Hamilton RL et al (2006) Chronic traumatic encephalopathy in a National Football League player: part II. Neurosurgery 59:1086-1092. https://doi.org/10.1227/01. NEU.0000245601.69451.27

Ommaya AK, Hirsch AE, Flamm ES, Mahone RH (1966) Cerebral concussion in the monkey: an experimental model. Science 153:211-212. https://doi.org/10.1126/science.153.3732.212

Pendergrass EP, Hodes PJ (1935) Dilatations of the cavum septi pellucidi and cavum vergæ. Ann Surg 101:269-295. https://doi. org/10.1097/00000658-193501000-00027 
Rosenthal JA, Foraker RE, Collins CL, Comstock RD (2014) National high school athlete concussion rates from 2005-2006 to 2011-2012. Am J Sports Med 42:1710-1715. https://doi. org/10.1177/0363546514530091

Rowson S, Beckwith JG, Chu JJ et al (2011) A six degree of freedom head acceleration measurement device for use in football. J Appl Biomech 27:8-14. https://doi.org/10.1123/jab.27.1.8

Rowson S, Duma SM, Beckwith JG et al (2012) Rotational head kinematics in football impacts: an injury risk function for concussion. Ann Biomed Eng 40:1-13. https://doi.org/10.1007/ s10439-011-0392-4

Sabet AA, Christoforou E, Zatlin B et al (2008) Deformation of the human brain induced by mild angular head acceleration. J Biomech 41:307-315. https://doi.org/10.1016/j.jbiom ech.2007.09.016

Schaltenbrand G (1975) The effects on speech and language of stereotactical stimulation in thalamus and corpus callosum. Brain Lang 2:70-77. https://doi.org/10.1016/S0093-934X(75)80055-1

Shenton ME, Hamoda HM, Schneiderman JS et al (2012) A review of magnetic resonance imaging and diffusion tensor imaging findings in mild traumatic brain injury. Brain Imaging Behav 6:137-192. https://doi.org/10.1007/s11682-012-9156-5

Siegmund GP, Guskiewicz KM, Marshall SW et al (2016) Laboratory validation of two wearable sensor systems for measuring head impact severity in football players. Ann Biomed Eng 44:12571274. https://doi.org/10.1007/s10439-015-1420-6

Smith DH (2015) Chronic neuropathologies of single and repetitive TBI: substrates of dementia? Nat Rev Neurol 9:211-221. https:// doi.org/10.1038/nrneurol.2013
Smith DH, Nonaka M, Miller R et al (2000) Immediate coma following inertial brain injury dependent on axonal damage in the brainstem. J Neurosurg 93:315-322. https://doi.org/10.3171/ jns.2000.93.2.0315

Sullivan S, Eucker SA, Gabrieli D et al (2015) White matter tractoriented deformation predicts traumatic axonal brain injury and reveals rotational direction-specific vulnerabilities. Biomech Model Mechanobiol 14:877-896. https://doi.org/10.1007/s1023 7-014-0643-z

Takhounts EG, Ridella SA, Hasija V et al (2008) Investigation of traumatic brain injuries using the next generation of simulated injury monitor (SIMon) finite element head model. Stapp Car Crash J 52:1-31. https://doi.org/10.4271/2008-22-0001

Ungerleider LG, Mishkin M (1982) Two cortical visual systems. In: Ingle DJ, Goodale MA, Mansfield RJW (eds) Analysis of visual behavior. MIT Press, Cambridge

Weaver AA, Danelson KA, Stitzel JD (2012) Modeling brain injury response for rotational velocities of varying directions and magnitudes. Ann Biomed Eng 40:2005-2018. https://doi.org/10.1007/ s10439-012-0553-0

Wu LC, Nangia V, Bui K et al (2016) In Vivo evaluation of wearable head impact sensors. Ann Biomed Eng 44:1234-1245. https://doi. org/10.1007/s10439-015-1423-3

Publisher's Note Springer Nature remains neutral with regard to jurisdictional claims in published maps and institutional affiliations

\section{Affiliations}

\section{Fidel Hernandez ${ }^{1}$. Chiara Giordano ${ }^{2} \cdot$ Maged Goubran $^{3} \cdot$ Sherveen Parivash $^{3} \cdot$ Gerald Grant $^{4,5} \cdot$ Michael Zeineh $^{3}$. David Camarillo ${ }^{1,2}$}

Fidel Hernandez

fidelh@alumni.stanford.edu

1 Department of Mechanical Engineering, Stanford University, Stanford, CA 94305, USA

2 Department of Bioengineering, Stanford University, Stanford, CA 94305, USA
3 Department of Radiology, Stanford University, Stanford, CA 94305, USA

4 Department of Neurosurgery, Stanford University, Stanford, CA 94305, USA

5 Department of Neurology, Stanford University, Stanford, CA 94305, USA 